\title{
Motor complications in an incident Parkinson's disease cohort
}

\begin{tabular}{|r|l|}
\hline Journal: & European Journal of Neurology \\
\hline Manuscript ID: & EJoN-15-0093.R1 \\
\hline Wiley - Manuscript type: & Original Papers \\
\hline Date Submitted by the Author: & n/a \\
\hline Complete List of Authors: & $\begin{array}{l}\text { Scott, Nicholas; University of Aberdeen, Division of Applied Health Sciences } \\
\text { Macleod, Angus; University of Aberdeen, Division of Applied Health } \\
\text { Sciences } \\
\text { Counsell, Carl; University of Aberdeen, Division of Applied Health } \\
\text { Sciences; }\end{array}$ \\
\hline Keywords: & Parkinson's disease < Movement disorders < NEUROLOGICAL DISORDERS \\
\hline
\end{tabular}

SCHOLARONE $^{\text {m }}$

Manuscripts 
1

2

3

4

5

6

7

8

10

11

12

13

14

15

16

17

18

19

20

21

22

23

24

25

26

27

28

29

30

31

32

33

34

35

36

37

38

39

40

41

42

43

44

45

46

47

48

49

50

51

52

53

54

55

56

57

58

59

60
Motor complications in an incident Parkinson's disease cohort

Nicholas W Scott ${ }^{1}$, Angus D Macleod ${ }^{1}$, Carl E Counsell ${ }^{1}$

Address: $\quad{ }^{1}$ Division of Applied Health Sciences

University of Aberdeen

Polwarth Building

Foresterhill

Aberdeen

AB25 2ZD

UK

Corresponding author: Dr. Angus D Macleod

Tel $+44(0) 1224437120$

Fax $+44(0) 1224437285$

email: angus.macleod@abdn.ac.uk

Keywords: $\quad$ Parkinson's disease

Motor fluctuations

Dyskinesias

Motor complications

Levodopa

Abstract word count: 249

Article word count: 3412

Running title: Motor complications in PD

Conflicts of Interest: We declare we have no conflicts of interest. 
ABSTRACT

Background

Levodopa treatment in Parkinson's disease (PD) causes motor fluctuations and dyskinesias, but few data describe their development or severity in unselected incident cohorts.

\section{Methods}

We gathered demographic, clinical, treatment, smoking, caffeine, and alcohol data from 183 people with PD from the PINE study, a community-based, incident cohort. With Kaplan-Meier survival analysis and Cox regression modelling we assessed the development, and severity, of dyskinesias and motor fluctuations and which factors independently influenced their onset.

\section{Results}

After mean follow-up of 59 months, 39 patients (21.3\%) developed motor fluctuations and 52 (28.4\%) developed dyskinesias. Kaplan-Meier estimates of the probability of motor fluctuations and dyskinesias after 5 years of dopaminergic treatment were $29.2 \%$ (95\% confidence interval [CI] 21.5$38.8 \%$ ) and $37.0 \%$ (95\% $\mathrm{Cl} 28.5-47.1 \%$ ) respectively. $19.8 \%$ developed motor fluctuations requiring treatment changes but only $4.0 \%(95 \% \mathrm{Cl} 1.5-10.4 \%)$ developed dyskinesias requiring treatment changes by five years. Cumulative levodopa dose (Hazard ratio [HR] 1.38 [95\% Cl 1.19-1.60]), female sex (HR 2.41 [1.19-4.89]), and younger age at diagnosis (HR 1.08 [1.04-1.11]) were independently associated with development of motor fluctuations. Cumulative levodopa dose (HR 1.23 [1.081.40]), female sex (HR 2.51 [1.40-4.51]) were independently associated with dyskinesias. In exploratory analyses, moderate caffeine exposure was associated with fewer motor fluctuations, longer symptom duration with more dyskinesias, and tremor at diagnosis with higher rates of both complications.

\section{Conclusions}

In this community-based incident PD cohort, severe dyskinesias were rare. Cumulative levodopa dose was the strongest predictor of both dyskinesias and motor fluctuations. 


\section{INTRODUCTION}

Treatment of Parkinson's disease (PD), especially with levodopa, causes dyskinesias and motor fluctuations. These motor complications are potentially disabling and may adversely affect patients' quality of life. ${ }^{1-3}$ A previous review of studies of motor complications estimated that the risk of developing motor fluctuations and dyskinesias were both about $40 \%$ after levodopa treatment for 4 6 years. ${ }^{4}$ However, most previous studies have been based on unrepresentative samples, with attendant selection biases, such as cohorts from specialist clinics or clinical trials in which younger patients with fewer co-morbidities than the general PD population are often over-represented. ${ }^{5,6}$ Only two representative, community-based incidence studies have examined the development of motor complications over time, both of which were small, only reported dyskinesias ${ }^{7,8}$ and one was retrospective. $^{8}$

Several risk factors for the development of dyskinesias have been identified, including: younger age at diagnosis, female sex, higher levodopa dose, longer duration of levodopa therapy, and lower body weight, $^{8-14}$ but most data come from non-incident or hospital-based studies. By contrast, few predictors of motor fluctuations have been identified but dose and duration on levodopa therapy are most commonly reported. ${ }^{12,15-18}$ In addition, nicotine, caffeine and alcohol may protect against the development of $\mathrm{PD}^{19}$ and there is some inconsistent clinical trial data to suggest caffeine and another adenosine $\mathrm{A} 2 \mathrm{~A}$ antagonist, may reduce dyskinesia risk. ${ }^{20,21}$

We therefore aimed to (i) describe the development of dyskinesias and motor fluctuations in a prospective, community-based, incident cohort of PD; (ii) assess what factors influence their development; and (iii) describe the development of severe motor complications. 


\section{METHODS}

\section{Study Design and Participants}

We used data from the Parkinsonism Incidence in North-East Scotland (PINE) study, a communitybased incidence study of PD and other parkinsonian disorders in Aberdeen and surrounding areas with prospective long-term follow-up. ${ }^{22,23}$ Attempts were made to identify all newly diagnosed patients with degenerative or vascular parkinsonism between 2002 and 2004 (pilot phase) and 2006 to 2009 (main study phase) using multiple, overlapping methods for case ascertainment. ${ }^{23}$ All patients were asked to consent to long-term annual follow-up with interim appointments as required for clinical management. The study was approved by the Multi-centre Research Ethics Committee for Scotland and conducted with the informed consent of the patients involved.

This analysis was restricted to patients who had a diagnosis of idiopathic PD at death or latest follow-up, made by a consultant neurologist with an interest in movement disorders (CEC) using the UK Brain Bank criteria, ${ }^{24}$ insofar as follow-up duration permitted supportive criteria to be applied. $15 \%$ of patients with a latest diagnosis of PD had initially had an alternative diagnosis. Patients were excluded if they were not followed-up after baseline or if they had not received dopaminergic treatment. Patients were treated according to the clinical judgement of the treating clinicians.

\section{Data collection and assessment of motor complications}

At the baseline (i.e. diagnostic) assessment and at subsequent follow-up appointments, consenting patients were interviewed and examined, and information gathered included patient demographics and clinical characteristics (including the Unified PD Rating Scale [UPDRS]) and details of parkinsonian medication. Data were also gathered on exposure to caffeine, alcohol and smoking at verbal interview, including age at first exposure, average level of exposure before baseline and, if relevant, year exposure stopped. At each annual assessment, patients were asked about their ongoing exposures. 
Data on motor complications were gathered from the prospectively acquired records of the examining study physician and from part IV of the UPDRS. In patients who reported motor complications, the month and year of onset were recorded from patient self-report or, in the case of asymptomatic dyskinesias that were seen at the assessment, the date of that assessment. Dyskinesias did not have to be witnessed by a physician to be included, but if they were not seen and the history was doubtful, they were not included. Severe motor complications were defined as those which required changes to parkinsonian treatment after discussion with the patient about their impact.

\section{Analysis}

The data were extracted from the PINE database $\left(26^{\text {th }}\right.$ June, 2013), checked and cleaned. The cumulative levodopa only dose and the total levodopa-equivalent dose (LED) ${ }^{25}$ (including any dopamine replacement therapy) were calculated up to four years from diagnosis since almost all surviving patients had been followed-up for four years. Levodopa and levodopa-equivalent doses were calculated as levodopa or levodopa-equivalent dose in milligrams multiplied by number of days of treatment and divided by $10^{5}$ to give units equivalent to about $70 \mathrm{mg}$ levodopa, on average, a day for 4 years. Cumulative alcohol and caffeine lifetime exposure were divided into tertiles and smoking exposure was categorised as never, low and high because there were large numbers of non-smokers in our cohort. Cups of tea and coffee were assumed to contain $47 \mathrm{mg}$ and $62 \mathrm{mg}$ of caffeine respectively. ${ }^{26}$ Tertiles of cumulative alcohol and caffeine exposure over three years after diagnosis were also calculated to investigate whether ongoing exposure after diagnosis was associated with fewer motor complications.

We performed Kaplan-Meier survival analysis of motor fluctuation-free and dyskinesia-free survival from the start of any dopamine replacement therapy (levodopa, dopamine agonist, MAO-B inhibitor) 
with patients censored at death or last follow-up. Survival analyses were also performed with different time baselines to facilitate comparisons with previous studies: i) levodopa initiation; and ii) diagnosis. Cox proportional hazards modelling was performed to assess what factors independently influenced the development of motor complications (using start of dopaminergic treatment as the baseline for survival). Univariable (unadjusted) hazards ratios were firstly calculated for all the variables listed in table 1 . There were too many variables to include all in a multivariable model. On the basis of a priori evidence we selected four variables to include (irrespective of statistical significance) in the two main Cox regression models (i.e. one predicting motor fluctuations and one dyskinesias): age at diagnosis, sex, motor UPDRS score at baseline and cumulative levodopa dose up to four years from diagnosis. This ensured no fewer than about 10 events per variable in the main models. ${ }^{27}$ Additional exploratory analyses were performed to investigate the role of other variables including symptom duration prior to diagnosis, weight, MMSE score at diagnosis, different treatment measures (cumulative levodopa-equivalent dose, starting levodopa within one year of diagnosis) and measures of smoking, alcohol and caffeine exposure. These secondary analyses were performed by creating models with the four pre-specified variables together with each of the additional variables in turn. There were no missing data in the variables used in the main analysis; in the secondary analysis, if missing data were present for a particular variable, these observations were excluded from analyses including that variable. Statistical analyses were performed using SPSS version 21 and Stata version 12

\section{RESULTS}

206 patients with a diagnosis of idiopathic PD at latest follow-up were identified from the PINE database (Figure 1), of which seven declined clinical follow-up, ten died before their first follow-up and six had not received dopaminergic medication by time of data extraction. These patients (mean age 70.8) were not treated because they had mild disease with tremor dominance $(\mathrm{N}=3)$, had early 
dementia while motor symptoms were still mild $(\mathrm{N}=1)$, refused treatment $(\mathrm{N}=1)$, or did not tolerate levodopa $(\mathrm{N}=1)$. The remaining 183 treated idiopathic PD patients $(57.4 \%$ male, mean age at diagnosis 71.7 years) were included in the main analyses. The mean duration of follow-up was 59 months (SD 22). 66 patients (36.1\%) died during the follow-up. Five patients (2.7\%) were lost to clinical follow-up. 128 patients (69.9\%) started levodopa within the first year of follow up (median duration to treatment onset 4 months, IQR 0-14 months). Most patients (69.9\%) received levodopa in the first year after diagnosis and a further $24(13.1 \%)$ received levodopa within four years of follow-up. The patients who received dopaminergic therapy in the first four years, but not levodopa, were mostly treated with ropinirole (33 patients), pramipexole (17 patients), selegiline (13 patients), COMT inhibitors ( 2 patients), rasagiline (1 patient), or ergot-derived dopamine agonists ( 2 patients).

\section{Motor Fluctuations}

Motor fluctuations occurred in 39 patients (21.3\%) (see table 1 and figure $2 \mathrm{~A}$ ). The majority of these were severe enough to require changes in therapy (25 patients [ $13.7 \%$ of total participants, $64.1 \%$ of those with motor fluctuations]). These changes were mostly increased frequency of levodopa dosage or adding controlled release levodopa at night. Kaplan-Meier estimates of probability of developing motor fluctuations at five years, with different baselines for time measurement, are shown in table 3. The factors independently associated with the development of motor fluctuations in the main multivariable model were higher cumulative levodopa dose, female sex, and younger age at diagnosis (Table 1). In secondary analyses the presence of tremor at diagnosis was associated with a higher rate and moderate (though not high) lifetime caffeine intake was associated with a lower rate of motor fluctuations.

Table 1. Characteristics of patients who developed motor fluctuations

\begin{tabular}{lllcc}
\hline Characteristics & Patients with & Patients & Unadjusted HR & Adjusted $\mathrm{HR}^{*}$ \\
& fluctuations & without & $(95 \% \mathrm{Cl})$ & $(95 \% \mathrm{Cl})$ \\
\hline
\end{tabular}




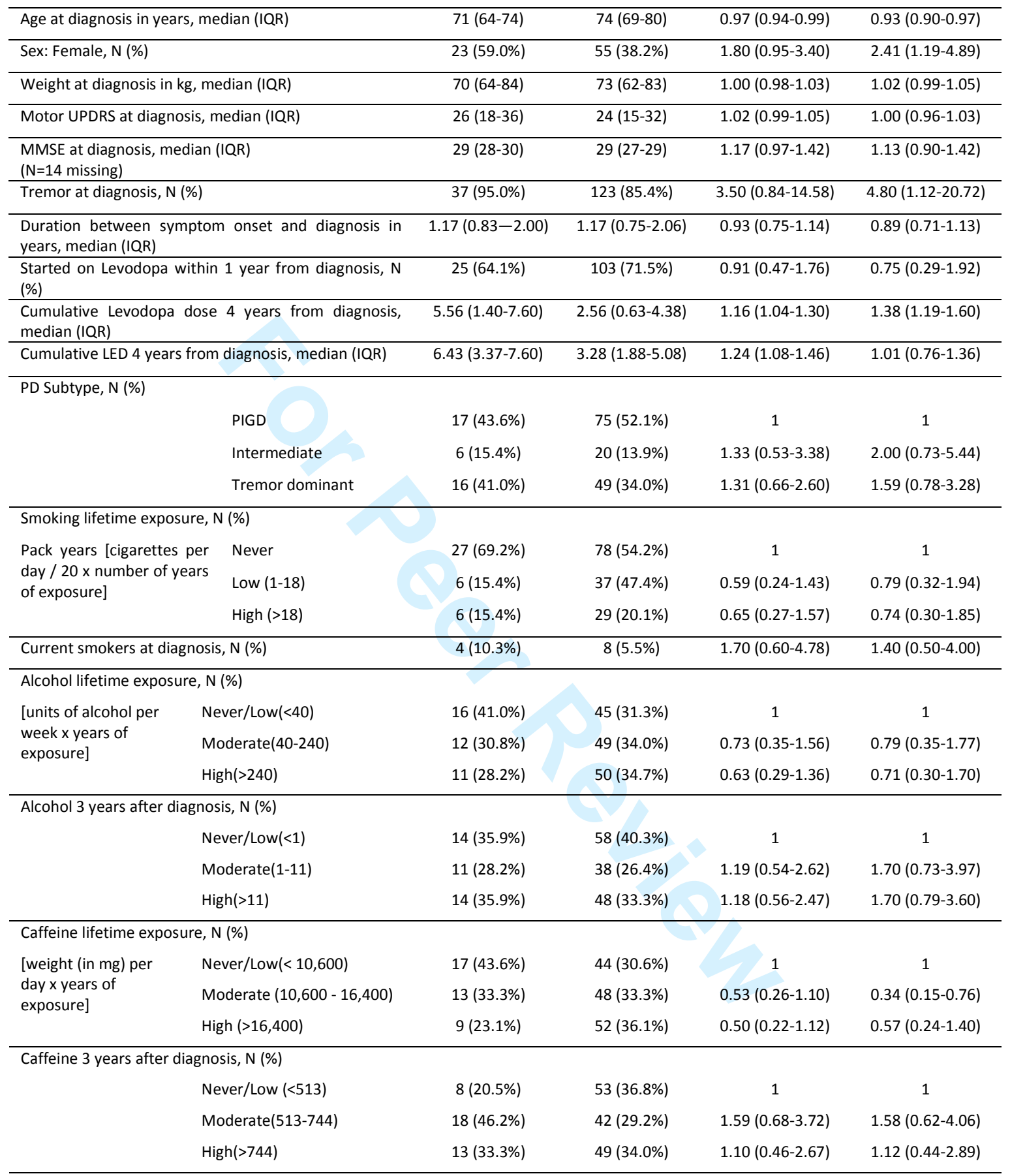

*Variables adjusted for the variables in the final multivariable model (age at diagnosis, sex, motor UPDRS at diagnosis, and cumulative levodopa dose 4). Abbreviation: PIGD= postural instability and gait disorder.

\section{Dyskinesias}

52 patients (28.4\%) developed dyskinesias (see table 2 and figure 2B). At onset, only three patients (1.6\% of total participants, $5.8 \%$ of those with dyskinesia) rated their dyskinesias as painful and five 
( $2.7 \%$ of total participants, $9.6 \%$ of those with dyskinesia) as mildly disabling; the rest were not disabling. Only 8 patients (4.4\% of total participants, $15.4 \%$ of those with dyskinesia) developed dyskinesias which required treatment changes (such as decrease of levodopa dose or addition of amantadine). The median dyskinesia-free survival time was 85 months. Kaplan-Meier estimates of the probability of developing dyskinesias after five years, with different baselines for time measurement, are shown in table 3. Higher cumulative levodopa dose and female sex were found to be independent risk factors for the development of dyskinesias in the main model (Table 2). In the additional models, symptom duration and the presence of tremor at diagnosis were associated with more dyskinesias and there was a suggestion that higher MMSE score was associated with increased risk.

Table 2. Characteristics of patients who developed dyskinesias

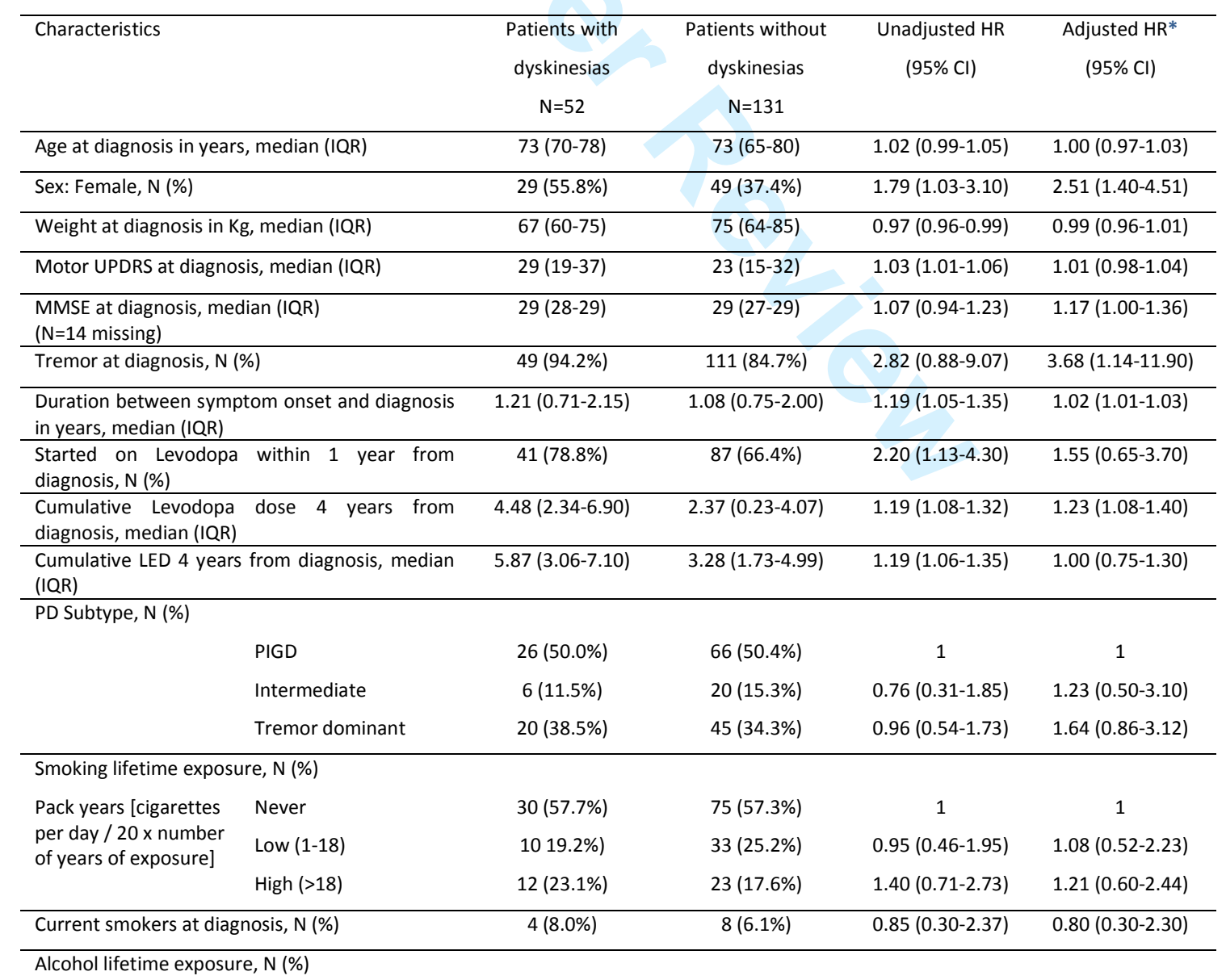




\begin{tabular}{|c|c|c|c|c|c|}
\hline \multirow{3}{*}{$\begin{array}{l}\text { [units of alcohol per } \\
\text { week } x \text { years of } \\
\text { exposure] }\end{array}$} & Never/Low $(<40)$ & $22(42.3 \%)$ & $39(29.8 \%)$ & 1 & 1 \\
\hline & Moderate(40-240) & $14(26.9 \%)$ & $47(35.9 \%)$ & $0.53(0.27-1.04)$ & $0.63(0.31-1.30)$ \\
\hline & $\operatorname{High}(>240)$ & $16(30.8 \%)$ & $45(34.4 \%)$ & $0.66(0.35-1.30)$ & $0.80(0.38-1.68)$ \\
\hline \multicolumn{6}{|c|}{ Alcohol 3 years after diagnosis, N (\%) } \\
\hline & Never/Low $(<1)$ & $21(40.4 \%)$ & $51(38.9 \%)$ & 1 & 1 \\
\hline & Moderate(1-11) & $11(21.2 \%)$ & $38(29.0 \%)$ & $0.67(0.32-1.39)$ & $0.90(0.42-1.92)$ \\
\hline & $\operatorname{High}(>11)$ & $20(38.5 \%)$ & $42(32.1 \%)$ & $1.09(0.59-2.01)$ & $1.63(0.85-3.14)$ \\
\hline \multicolumn{6}{|c|}{ Caffeine lifetime exposure, N (\%) } \\
\hline \multirow{3}{*}{$\begin{array}{l}\text { [weight (in } \mathrm{mg} \text { ) per } \\
\text { day } x \text { years of } \\
\text { exposure] }\end{array}$} & Never/Low $(<10,600)$ & $16(30.7 \%)$ & $45(34.4 \%)$ & 1 & 1 \\
\hline & $\begin{array}{l}\text { Moderate }(10,600 \text { - } \\
16,400)\end{array}$ & $20(38.5 \%)$ & $41(31.3 \%)$ & $1.26(0.65-2.43)$ & $0.81(0.40-1.64)$ \\
\hline & High $(>16,400)$ & $16(30.7 \%)$ & $45(34.4 \%)$ & $1.13(0.56-2.25)$ & $0.80(0.38-1.67)$ \\
\hline \multicolumn{6}{|c|}{ Caffeine 3 years after diagnosis, N (\%) } \\
\hline & Never/Low $(<513)$ & $11(21.2 \%)$ & $50(38.2 \%)$ & 1 & 1 \\
\hline & Moderate(513-744) & $20(38.5 \%)$ & 40 (30.5\%) & $1.56(0.74-3.29)$ & $1.40(0.64-3.08)$ \\
\hline & High(>744) & $21(40.4 \%)$ & $41(31.3 \%)$ & $1.56(0.75-3.24)$ & $1.37(0.65-2.87)$ \\
\hline
\end{tabular}

*Variables are adjusted for the variables in the final multivariable model (age at diagnosis, sex, motor UPDRS at diagnosis, and cumulative levodopa dose). Abbreviation: PIGD= postural instability and gait disorder.

Table 3: Kaplan-Meier probabilities of developing motor fluctuations and dyskinesias after five years from dopaminergic treatment initiation, from levodopa initiation, and from diagnosis.

\begin{tabular}{llr}
\hline Baseline for time measurement & Motor fluctuations & Dyskinesias \\
\hline Any complication & & \\
\hline Starting dopaminergic treatment $(\mathrm{N}=183)$ & $29.2 \%(21.5-38.8)$ & $37.0 \%(28.5-47.1)$ \\
Starting levodopa treatment $(\mathrm{N}=160)$ & $30.6 \%(22.6-40.7)$ & $43.6 \%(33.7-54.9)$ \\
$\quad$ Diagnosis ( $\mathrm{N}=189)$ & $22.8 \%(16.7-30.7)$ & $29.6 \%(22.7-37.8)$ \\
\hline Severe complications & & $4.0 \%(1.5-10.4)$ \\
\hline Starting dopaminergic treatment $(\mathrm{N}=183)$ & $19.8 \%(13.4-28.8)$ & \\
\hline 95\% confidence intervals are in parentheses. & &
\end{tabular}

\section{Motor fluctuations and dyskinesias}

23 patients (12.6\%) developed both fluctuations and dyskinesias, of whom 14 had developed dyskinesias first.

\section{DISCUSSION}


About $30 \%$ of patients with PD developed motor fluctuations and about $37 \%$ developed dyskinesias within five years of starting dopaminergic treatment. These were higher risks than described in two other community-based incidence cohorts that examined the development of dyskinesias. A Mayo Clinic series reported that $30 \%$ of patients developed dyskinesias by five years of starting levodopa ${ }^{8}$ and in another study fewer than $20 \%$ of patients developed dyskinesias five years from diagnosis. ${ }^{7}$ The reasons for these differences, between similar studies, are unclear. No previous communitybased inception studies have reported the frequency of motor fluctuations. We found complications to be less common than several other studies, some of which were older and therefore used higher levodopa doses than are used in current practice. ${ }^{4,18}$ Dyskinesias were more common in our cohort than motor fluctuations, similar to some studies ${ }^{15,28}$ but not others. ${ }^{16,12,18}$ Disabling dyskinesias were rare and, although most patients with motor fluctuations did need changes in treatment, very few (4\%) developed dyskinesias necessitating treatment changes. This is lower than one previous incident study in which $17 \%$ of patients required treatment changes for dyskinesias within five years of levodopa initiation. ${ }^{8}$ That study used patient records between 1976 and 1990 and the lower incidence of severe dyskinesias in our study, could be due to lower levodopa doses used in more recent prescribing practice.

Levodopa exposure has consistently been reported as the strongest risk factor for the development of motor fluctuations and dyskinesias in observational studies, ${ }^{4,8,16}$ randomised trials of levodopa versus dopamine agonists ${ }^{29,30}$ and randomised trials of different levodopa doses. ${ }^{31}$ Previous studies have investigated several measures of levodopa exposure, including the initial average daily dose, ${ }^{7}$ average daily LED; ${ }^{8}$ or levodopa dose at onset of dyskinesias (or study end in those without dyskinesias). ${ }^{12}$ Here we have compared cumulative both levodopa only and levodopa-equivalent doses up to four years and early (within one year of diagnosis) commencement of levodopa. Although these variables were correlated, only cumulative levodopa dose was significantly associated with motor complications when they were entered into a multivariable model together. 
This suggests that there is little additional effect of non-levodopa dopaminergic treatments, similar to findings from a recent meta-analysis. ${ }^{32}$ It also suggests there is no absolute requirement to avoid early levodopa treatment in PD but, as others have suggested, ${ }^{12}$ it is important to use the smallest dose that adequately controls the patient's symptoms throughout the course of PD. The fact that baseline motor severity (UPDRS) was not an independent risk factor for motor complications suggests that the association between cumulative levodopa dose and motor complications is not just due to worse disease severity with greater dopaminergic neuronal loss in those needing more levodopa. This conflicts with one large trial which found that both baseline disease severity and levodopa doses were associated with higher frequency of motor complications. ${ }^{12}$ However, evidence that levodopa increases risk of dyskinesias independently of disease severity also comes from randomised clinical trials, in which disease severity is randomly allocated to each arm, and those with higher doses of levodopa ${ }^{31}$ or levodopa and entacapone ${ }^{33}$ had higher incidence of dyskinesias.

Many previous studies have shown that younger age at onset is associated with more dyskinesias, ${ }^{9,10}$ and it was the most powerful predictor in a recent large trial. ${ }^{12}$ We found that age at diagnosis did not predict dyskinesias, similar to another community-based incidence study. ${ }^{7}$ It may be that, as a community-based incidence study, with proportionally few young-onset patients (4.4\% under 50), there was insufficient power to detect an effect of age on dyskinesias.

Duration between symptom onset and diagnosis was found to be an independent risk factor for developing dyskinesias but not motor fluctuations. This result was independent of baseline disease severity and levodopa dose so does not appear to be an effect of those presenting later being treated with higher doses of levodopa. This finding must be interpreted cautiously because it was a secondary analysis and the variable is subject to recall bias but it may suggest that patients with more indolent onset of their PD symptoms may be at a higher risk of developing dyskinesias. 
The presence of tremor at diagnosis was associated with higher risk of both motor fluctuations and dyskinesias in the secondary analyses, which contrasts with a previous smaller study. ${ }^{34}$ Although consistent for both types of complications, the number without any tremor at baseline in this posthoc analysis was small so this finding, whilst novel and interesting, requires replication.

Female gender was an independent risk factor for both motor fluctuations and dyskinesias, as previous studies have also shown. ${ }^{12}$ The reasons for gender differences in the development of motor complications are unclear. A possible explanation is that lower average weight in females results in higher levodopa doses per body weight, and some previous studies found lower weight was a risk factor for dyskinesias. ${ }^{35,36}$ We did not, however, demonstrate an association with baseline weight but changes in weight after diagnosis may be more important in the development of complications. It has also been suggested that females have a reduced genetic protection from a dopamine receptor polymorphism, ${ }^{11}$ and hormonal differences may be important, with evidence from animal models of effects of oestrogen on the basal ganglia. ${ }^{37}$

Moderate lifetime caffeine exposure was associated with a reduced risk of developing motor fluctuations in the secondary multivariable analyses but there was no dose-response gradient and, given the large number of associations tested in the secondary analyses, it may well be a false positive. Nevertheless, a clinical trial showed caffeine lowered risk of dyskinesias ${ }^{20}$ and a trial of another adenosine A2A antagonist, istradefylline reduced daily OFF time. ${ }^{21}$ We did not find smoking or alcohol exposure, either before or after diagnosis to be associated with lower risk of dyskinesias. This is consistent with a previous study which showed smoking was not associated with motor complications. ${ }^{14}$ However, we lacked power to identify small effects of these exposures on motor complications. 
The principal strengths of this study are its prospective design; the representative sample (attempts were made to identify all patients in the community with a new parkinsonian syndrome); regular reviewed of diagnoses to improve diagnostic accuracy; frequent (at least yearly) clinical review to obtain data on motor complications; very high study retention; and careful statistical analyses. Additionally, cumulative levodopa doses were calculated up to 4 years rather than at complication onset. This is clearly better than comparing levodopa dose at onset of motor complications with levodopa dose at end of study in those without complications as this is confounded by difference in time.

The study has several limitations. Firstly, study size, while not small in terms of previous studies of motor complications, is insufficient to identify weak associations or investigate interactions. Secondly, average follow-up duration was only about five years, so better data may be obtained with longer follow-up. Thirdly, some inaccuracy in defining onset of complications is inevitable. Exact timing of onset of both dyskinesias and motor fluctuations was mostly subject to patient recall, although some patients' dyskinesias were observed at clinic visits before they were noticed by the patients themselves, and were recorded as starting when seen. Thus the time to onset of dyskinesias may be overestimated. Assessment of severity was based on data about changes in therapy, which was derived from comprehensive clinical letter that invariably included reasons for treatment changes so we believe this was a reliable assessment. Fourthly, the secondary analyses must be considered as exploratory as many variables were examined and type I errors are possible. Fifthly, data on caffeine, smoking and alcohol were partly retrospective, only average exposures were used, and we did not gather data on sources of caffeine other than tea and coffee.

In conclusion, we are the first to describe the development of both motor fluctuations and dyskinesias in a representative, community-based, incident cohort of PD. We estimate that $29 \%$ and $37 \%$ develop motor fluctuations and dyskinesias respectively after 5 years of dopaminergic 
treatment. Dyskinesias requiring treatment changes were rare ( $4 \%$ at 5 years), which is lower than previous estimates. Higher cumulative levodopa dose, female sex, and tremor at diagnosis were independent risk factors for both motor complications; moderate lifetime caffeine exposure and younger age for fewer motor fluctuations; and longer pre-diagnosis symptom duration for more dyskinesias. Further work with more patients with longer follow-up would be useful for more detailed analysis of risk factors. Individual-patient-data meta-analysis of existing representative studies would be an efficient way to do this.

\section{ACKNOWLEDGEMENTS}

We acknowledge funding for the PINE study from Parkinson's UK, the Scottish Chief Scientist Office, the BMA Doris Hillier award, RS Macdonald Trust, the BUPA Foundation, NHS Grampian endowments and SPRING. We thank the patients for their participation and the research staff who collected data and supported the study database.

\section{AUTHOR'S ROLES}

The authors contributed to the following specific roles in the project and manuscript preparation as indicated:

1. Research project: A. Conception, B. Organization, C. Execution;

2. Statistical Analysis: A. Design, B. Execution, C. Review and Critique;

3. Manuscript Preparation: A. Writing of the first draft, B. Review and Critique

Nicholas W Scott (1B, 1C, 2B, 2C, 3A)

Angus D Macleod (1B, 2A, 2B, 2C, 3B)

Carl E Counsell (1A, 1B, 2C, 3B) 


\section{FINANCIAL DISCLOSURE}

Nicholas W Scott: none

Angus D Macleod: Funded by a Clinical Academic Fellowship from the Scottish Chief Scientist Office. Also received research funding from Parkinson's UK.

Carl E Counsell: Research funding from Parkinson's UK, Scottish Chief Scientist Office, National Institute of Health Research, and Engineering and Physical Sciences Research Council

\section{REFRENCES}

1. Samii A, Nutt JG, Ransom BR. Parkinson's disease. Lancet 2004; 363 (9423):1783-1794.

2. Chapuis S, Ouchchane L, Metz O, Gerbaud L, Durif F. Impact of the motor complications of Parkinson's disease on the quality of life. Movement Disorders 2005; 20 (2): 224-230.

3. Marras C, Lang A, Krahn M, Tomlinson G, Naglie G. Parkinson Study Group, Quality of life in early Parkinson's disease. Movement Disorders 2004; 19 (1): 22-28.

4. Ahlskog JE, Muenter MD. Frequency of levodopa-related dyskinesias and motor fluctuations as estimated from the cumulative literature. Movement Disorders 2001; 16 (3): 448-458.

5. Rybicki BA, Johnson CC, Gorell JM. Demographic differences in referral rates to neurologists of patients with suspected Parkinson's disease: implications for case-control study design. Neuroepidemiology 1995; 14 (2): 72-81.

6. Fitzsimmons PR, Blayney S, Mina-Corkill S, Scott GO. Older participants are frequently excluded from Parkinson's disease research. Parkinsonism Related Disorders 2012; 18 (5): 585-589. 
7. Evans JR, Mason SL, Williams-Gray CH, et al. The natural history of treated Parkinson's disease in an incident, community based cohort. J Neurology Neurosurgery Psychiatry 2011; 82 (10): 11121118.

8. Van Gerpen JA, Kumar N, Bower JH, Weigand S, Ahlskog JE. Levodopa-associated dyskinesia risk among Parkinson disease patients in Olmsted County, Minnesota, 1976-1990. Archives Neurology 2006; 63 (2): 205-209.

9. Kumar N, Van Gerpen JA, Bower JH, Ahlskog JE. Levodopa-dyskinesia incidence by age of Parkinson's disease onset. Movement Disorders 2005; 20 (3): 342-434.

10. Ku S, Glass GA. Age of Parkinson's disease onset as a predictor for the development of dyskinesia. Movement Disorders 2010; 25 (9): 1177-1182.

11. Zappia M, Annesi G, Nicoletti G, et al. Sex Differences in clinical and genetic determinants of levodopa peak-dose dyskinesias in Parkinson disease: an exploratory study. Archives Neurology 2005; 62 (4): 601-605.

12. Olanow CW, Kieburtz K, Rascol O, et al. Factors Predictive of the Development of LevodopaInduced Dyskinesia and Wearing-Off in Parkinson's Disease. Movement Disorders 2013; 28 (8): $1064-1071$.

13. Rascol O, Brooks DJ, Korczyn AD, et al. Development of dyskinesias in a 5-year trial and ropinirole and L-dopa. Movement Disorders 2006; 21 (11): 1844-1850.

14. Hassin-Baer S, Molchadski I, Cohen OS, et al. Gender effect on time to levodopa-induced dyskinesias. Journal of Neurology 2011; 258 (11): 2048-2053.

15. Garcia-Ruiz PJ, del Val J, Fernandez IM, Herranz A. What factors influence motor complications in Parkinson disease?: A 10-year prospective study. Clinical Neuropharmacology 2012; 35 (1):1-5.

16. Schrag A, Quinn N. Dyskinesias and motor fluctuations in Parkinson's disease: a communitybased study. Brain 2000; 123 (11): 2297-2305.

17. Jankovic J. Motor fluctuations and dyskinesias in Parkinson's disease: clinical manifestations. Movement Disorders 2005; 20 (Suppl. 11): S11-16. 
18. Hauser RA, McDermott MP, Messing S. Factors associated with the development of motor fluctuations and dyskinesias in Parkinson disease. Archives Neurology 2006; 63 (12): 1756-1760.

19. Noyce AJ, Bestwick JP, Silveira-Moriyama, et al. Meta-analysis of early nonmotor features and risk factors for Parkinson disease. Annals Neurology 2012; 72 (6): 893-901.

20. Wills AM, Eberly S, Tennis M, et al. Caffeine consumption and risk of dyskinesia in CALM-PD. Movement Disorders 2013; 28 (3): 380-383.

21. Mizuno $\mathrm{Y}$, Kondo $\mathrm{T}$, Japanese Istradefylline study group. Adenosine A2a receptor antagonist Istradefylline reduces daily OFF time in Parkinson's disease. Movement Disorders 2013; 28 (8): $1138-1141$.

22. Taylor KS, Counsell CE, Harris CE, Gordon JC, Smith WC. Pilot study of the incidence and prognosis of degenerative Parkinsonian disorders in Aberdeen, United Kingdom: methods and preliminary results. Movement Disorders 2006; 21 (7): 976-982.

23. Caslake R, Scott N, Gordon J, et al. Age-, gender-, and socioeconomic status-specific incidence of Parkinson's disease and parkinsonism in North East Scotland: The PINE study. Parkinsonism Related Disorders 2013; 19 (5): 515-521.

24. Hughes AJ, Daniel SE, Kilford L, Lees AJ. Accuracy of clinical diagnosis of idiopathic Parkinson's disease: a clinico-pathological study of 100 cases. J Neurology Neurosurgery Psychiatry 1992; 55 (3): 181-184.

25. Tomlinson CL, Stowe R, Patel S, Rick C, Gray R, Clarke CE. Systematic review of levodopa dose equivalency reporting in Parkinson's disease. Movement Disorders 2010: 25 (15): 2649-2653.

26. U.S. Department of Agriculture, Agriculture Research Service. USDA National Nutrient Database for Standard Reference, Release 24. 2011.

27. Peduzzi P, Concato J, Feinstein AR, Holford TR. Importance of events per independent variable in proportional hazards regression analysis, II: accuracy and precision of regression estimates. Journal of Clinical Epidemiology 1995; 48 (12): 1503-1510. 
28. Mazzella L, Yahr MD, Marinelli L, Huang N, Moshier E, Di Rocco A. Dyskinesias predict the onset of motor response fluctuations in patients with Parkinson's disease on I-dopa monotherapy, Parkinsonism Related Disorders 2005; 11 (3): 151-155.

29. Parkinson study Group. Pramipexole vs levodopa as initial treatment for Parkinson's disease. A randomised controlled trial. JAMA 2000; 284 (15): 1931-1938.

30. Parkinson Study Group CALM Cohort Investigators. Long-term effect of initiating pramipexole vs levodopa in early Parkinson disease. Archives Neurology 2009; 66 (5): 563-570.

31. Fahn S, Oakes D, Shoulson I, et al. Levodopa and the progression of Parkinson's disease. New England Journal of Medicine 2004; 351 (24): 2498-2508.

32. Chondrogiorgi M, Tatsioni A, Reichmann $\mathrm{H}$ and Konitsiotis S. Dopamine agonist monotherapy in Parkinson's disease and potential risk factors for dyskinesia: a meta-analysis of levodopa controlled trials. European Journal of Neurology 2014; 21 (3): 433-440.

33. Stocchi F, Rascol O, Kieburtz K, et al. Initiating levodopa/carbidopa therapy with and without entacapone in early Parkinson disease. The STRIDE-PD study. Annals of Neurology 2010; 68 (1): 18-27.

34. Kipfer S, Stephan MA, Schüpbach WM, Ballinari P, Kaelin-Lang A. Resting tremor in Parkinson disease: a negative predictor of levodopa-induced dyskinesia. Archives Neurology 2011; 68 (8): 1037-1039.

35. Sharma JC, Macnamara L, Hasoon M, Vassallo M, Ross IN. Cascade of levodopa dose and weight related dyskinesia in Parkinson's disease (LD-WD-PD cascade). Parkinsonism Related Disorders 2006; 12 (8): 499-505.

36. Sharma JC, Ross IN, Rascol O, Brooks D. Relationship between weight, levodopa and dyskinesia: the significance of levodopa dose per kilogram body weight. European Journal of Neurology 2008; 15 (5): 493-496.

37. Smith KM, Dahodwala, N. Sex differences in Parkinson's disease and other movement disorders. Experimental Neurology 2014; 259: 44-56. 


\title{
FIGURE LEGENDS
}

\author{
Figure 1. Flow chart of patients included in the analysis and initiation of levodopa treatment. FU: Follow-Up. \\ *Patients received only dopamine agonist or MAO-B inhibitors within 4 years of follow-up, except for 5 \\ patients who received treatment after 4 years of follow-up.
}

Figure 2. Kaplan-Meier estimates of the probability that PD patients on a dopaminergic therapy will be free from (A) motor fluctuations and (B) dyskinesias. In each graph, the blue line represents the development of any motor complication and the red represents the development of severe complications, i.e., those needing changes to treatment. 
1

2

3

6

Motor complications in an incident Parkinson's disease cohort

Nicholas W Scott ${ }^{1}$, Angus D Macleod ${ }^{1}$, Carl E Counsell ${ }^{1}$

\author{
Address: $\quad{ }^{1}$ Division of Applied Health Sciences \\ University of Aberdeen \\ Polwarth Building \\ Foresterhill \\ Aberdeen \\ AB25 2ZD \\ UK \\ Corresponding author: Dr. Angus D Macleod \\ Tel $+44(0) 1224437120$ \\ Fax $+44(0) 1224437285$ \\ email: angus.macleod@abdn.ac.uk \\ Keywords: \\ Parkinson's disease \\ Motor fluctuations \\ Dyskinesias \\ Motor complications \\ Levodopa
}

\begin{abstract}
word count: 249
Article word count: 3412
\end{abstract}

Running title: Motor complications in PD

Conflicts of Interest: We declare we have no conflicts of interest. 


\begin{abstract}
Background

Levodopa treatment in Parkinson's disease (PD) causes motor fluctuations and dyskinesias, but few data describe their development or severity in unselected incident cohorts.
\end{abstract}

\title{
Methods
}

We gathered data on-demographics, clinical-details, drug treatment ${ }_{2}$ and smoking, caffeine $_{2}$ and alcohol data history from 183 people with PD from the PINE study, a community-based, incident cohort. With Kaplan-Meier survival analysis and Cox regression modelling we assessed the development, and severity, of dyskinesias and motor fluctuations and which factors independently influenced their onset.

\section{Results}

After mean follow-up of 59 months, 39 patients (21.3\%) developed motor fluctuations and 52 (28.4\%) developed dyskinesias 59 manths (SD). Kaplan-Meier estimates of the probability of motor fluctuations and dyskinesias after 5 years of dopaminergic treatment $29.2 \%$ (95\% confidence interval $[\mathrm{Cl}] 21.5-38.8 \%)$ and ef dyskinesias was-37.0\% (95\% $\mathrm{Cl} 28.5-47.1 \%)$ respectively. $19.8 \%$ developed motor fluctuations requiring treatment changes but only $4.0 \%$ (95\% Cl 1.5-10.4\%) developed dyskinesias requiring treatment changes by five years. Cumulative levodopa dose (Hazard ratio [HR] 1.38 [95\% Cl 1.19-1.60]), female sex (HR 2.41 [1.19-4.89]), and, and younger age at diagnosis (HR 1.08 [1.04-1.11])_-were independently associated with development of motor fluctuations. Cumulative levodopa dose (HR 1.23 [1.08-1.40]), and female sex (HR 2.51 [1.40-4.51]) were independently associated with dyskinesias. In mondary-exploratory analyses, moderate caffeine exposure was associated with rates offewer motor fluctuations, longer symptom duration with more dyskinesias, and tremor at diagnosis with higher rates of both complications.

\section{Conclusions}

In this community-based incident PD cohort, severe dyskinesias were rare. Cumulative levodopa dose was the strongest predictor of both dyskinesias and motor fluctuations. 


\section{INTRODUCTION}

Treatment of Parkinson's disease (PD), especially with levodopa, is associated with the developmentcauses of motor complications, namely dyskinesias and motor fluctuations. These motor complications are common and-potentially disabling consequences of chronic levodopa therapyand may adversely affect patients' quality of life..$^{1-3}$

Ahlskgg and Muenter revied_A previous review of studies of dyskinesias and-motor fluctuationscomplications and-estimated that the risk of developing motor fluctuations and dyskinesias were both about $40 \%$ after levodopa treatment for 4-6 years. ${ }^{4}$ However, most previous studies have been based on unrepresentative samples, with attendant selection biases, such as cohorts derived-from specialist clinics eohortsor clinical trials in which younger enset-patients with fewer co-morbidities than the general PD population are often over-represented. ${ }_{-}^{5_{2}}$-or from clinical trials in which trial participants again tend to beare, on average, vounger than the general $P Q$ population with fewer co-morbidities. ${ }^{6}$ Only two representative, community-based incidence studies have examined the development of motor complications over time, both of which were small, only reported dyskinesias ${ }^{7,8}$ and one was retrospective. ${ }^{8}$ The latter show that whilst dyskinesias were common $(30 \%)$ at five years, most were mild and only $17 \%$ required treatment adjustment. ${ }^{8}$

Several risk factors for the development of dyskinesias have been identified, including: younger age at PD-diagnosis, female sex, higher levodopa dose, longer duration of levodopa therapy, and lower body weight ${ }_{-2}^{8-14}$ The two previous community-based incident cohorts both found that higher initial tevodopa dose was an independent risk factor for developing dyskinesias, whereas only one ${ }^{8}$ found age at diagnosis to be an independent risk factorbut most data come from non-incident or hospitalbased studies. By contrast, elatively few on predictors of motor fluctuations are been identified but dose and duration on levodopa therapy are most commonly reported. ${ }^{12,15-18}$ In 
addition, eigarette smokingnicotine, caffeine and alcohol may protect against the development of $\mathrm{PD}^{19}$ and there is also-some inconsistent clinical trial dataevidence from clinical trials to suggest that caffeine and another adenosine A2A antagonist, may reduce dyskinesiathe risk-of developing motor complications. ${ }^{20,21}$

We therefore aimed to (i) describe the development of dyskinesias and motor fluctuations in a prospective, community-based, incident cohort of development; and (iii) describe the development of severe dyskinesias and-motor fluctuationscomplications.

\section{METHODS}

\section{Study Design and Participants}

We used data from the Parkinsonism Incidence in North-East Scotland (PINE) study, a communitybased incidence study of PD and other parkinsoniansm disorders in Aberdeen and surrounding areas with prospective long-term follow-up. ${ }^{22,23}$ Attempts were made to identify all newly diagnosed patients with degenerative or vascular parkinsonism between 2002 and 2004 (pilot phase) and 2006 to 2009 (main study phase) using multiple, overlapping methods for case ascertainment. ${ }^{23}$ All patients were asked to consent to long-term annual follow-up with interim appointments as required for clinical management. The PINE-study was approved by the Multi-centre Research Ethics Committee for Scotland and was-conducted with the informed consent of the patients involved.

This analysis was restricted to patients who had a diagnosis of idiopathic PD at death or latest follow-up, made by a consultant neurologist with an interest in movement disorders (CEC) using the UK Brain Bank criteria, ${ }^{24}$ insofar as follow-up duration permitted the-supportive criteria to be applied. $15 \%$ of patients with a latest diagnosis of PD had initially had an alternative diagnosis. 
Patients were excluded if they were not followed-up after baseline or if they had not received dopaminergic treatment. The study size was determined by the number of incident patients in the study period. Treatment was initiated and managed Patients were treated according to the clinical judgement of the treating clinicians.

\section{Data collection and assessment of motor complications}

At the baseline (i.e. diagnostic) assessment and at subsequent follow-up appointments, consenting patients had consented were interviewed and examined, and information gathered included patient demographics and clinical characteristics (including the Unified PD Rating Scale [UPDRS]) and detains of parkinsonian medication. Data were also gathered on exposure to caffeine, alcohol and smoking at verbal interview, including age at first exposure, average level of exposure before baseline and, if relevant, year exposure stopped. At each annual assessment, patients were asked about their ongoing exposures.

Data on motor complications were gathered from the prospectively acquired records of the examining study physician and from part IV of the UPDRS. In patients who reported motor complications, the month and year of onset were recorded from patient self-report or, in the case of asymptomatic dyskinesias that were seen at the assessment, the date of that assessment. (which were based on clinical history and examination and included date of onset) and from part IV of the UPDRS. Dyskinesias did not have to be witnessed by a physician to be included, but if they were not seen and the history was doubtful, they were not included. Severe motor complications were defined as those which requireding changes to parkinsonianin treatment after discussion with the patient about their impact.

\section{Analysis}


The data were extracted from the PINE database $\left(26^{\text {th }}\right.$ June, 2013), checked and cleaned. histories were updated each year, from which we calculated $t$ The cumulative levodopa only dose and the total levodopa-equivalent dose (LED) ${ }^{25}$ (including any dopamine replacement therapy) were calculated up to four years from diagnosis since almost all surviving patients had been followed-up for four years. Levodopa and levodopa-equivalent doses were calculated as levodopa or levodopaequivalent dose in milligrams multiplied by number of days of treatment and divided by $10^{5}$ to give units equivalent to about 70mg levodopa, on average, a day for 4 years. Heasures of cumulative lifetime exposure at baseline for caffeine, alcohol and smoking were calculated.Cumulative Aalcohol and caffeine lifetime exposure as never, low and high because there were large numbers of non-smokers in our cohort. Weight of Eaffeine was calculated on the basis of a $\in \underline{C}$ ups of tea and coffee were assumed to containing $47 \mathrm{mg}$ and $62 \mathrm{mg}$ of caffeine respectivelyand a cup of coffee containing $62 \mathrm{mg} .{ }^{26}$ Aeasures-Tertiles of cumulative alcohol and caffeine exposure to caffeine and alcohol-over three years after diagnosis were also calculated to investigate whether ongoing exposure after diagnosis was associated with fewer motor complications-and divided into tertiles; this was not calculated for smoking as there were too few current smokers in the study.

We performed Kaplan-Meier survival analysis of motor fluctuation-free and dyskinesia-free survival from the start of any dopamine replacement therapy (levodopa, dopamine agonist, MAO-B inhibitor) with patients censored at death or last follow-up. Survival analyses were also performed with different time baselines to facilitate comparisons with previous studies: i) levodopa initiation; and ii) diagnosis. Cox proportional hazards modelling was performed to assess what factors independently influenced the development of motor complications from the onset of dopaminergic therapy (using start of dopaminergic treatment as the baseline for survival). Univariable (unadjusted) hazards ratios were firstly calculated for all the variables listed in table 1 . There were too many variables to include all in a multivariable model. On the basis of a priori evidence we selected four variables to include 


\section{RESULTS}

$21 \underline{2} \underline{06}$ patients with a diagnosis of idiopathic PD at latest follow-up were identified from the PINE database (Figure 1), of which 11 seven had-declined clinical follow-up, a further-ten had-died before their first follow-up and eightsix had not received any-dopaminergic medication by time of data extraction. These patients (mean age 70.8) were not treated because they had mild disease with tremor dominance $(\mathrm{N}=3)$, had early dementia while motor symptoms were still mild $(\mathrm{N}=1)$, refused treatment $(\mathrm{N}=1)$, or did not tolerate levodopa $(\mathrm{N}=1)$. The remaining 183 treated idiopathic PD patients ( $57.4 \%$ male, mean age at diagnosis 71.7 years) were included in the main analyses. The mean duration of follow-up was 59 months (SD 22). 66 patients (36.1\%) died during the follow-up. Five patients (2.7\%) were lost to clinical follow-up. 128 patients $(69.9 \%)$ started levodopa within the first year of follow up (median duration to treatment onset 4 months, IQR 0-14 months). Most 
patients (69.9\%) received levodopa in the first year after diagnosis and a further 24 (13.1\%) received levodopa within four years of follow-up. The patients who received dopaminergic therapy in the first four years, but not levodopa, were mostly treated with ropinirole (33 patients), pramipexole (17 patients), selegiline (13 patients), COMT inhibitors ( 2 patients), rasagiline ( 1 patient), or ergotderived dopamine agonists ( 2 patients).

\section{Motor Fluctuations}

Motor fluctuations occurred in 39 patients (21.3\%)-by the time of dable 1 and figure $2 \mathrm{~A}$ ). The majority of these were severe enough to require changes in therapy ( 25 patients [13.7\% of total participants, $64.1 \%$ of those with motor fluctuations]). These changes were mostly increased frequency of levodopa dosage or adding a-controlled release levodopa at night. KaplanMeier estimates of probability of developing motor fluctuations at five years, with different baselines for time measurement, are shown in table 3. After five years on therapy the Kaplan-Meier estimate of the probability of developing any motor fluctuations was $29.2 \%(95 \% \mathrm{Cl} 21.5-38.8 \%)$ and of developing severe motor fluctuations was $19.8 \%(95 \% \mathrm{Cl} 13.4-28.8 \%)$ (Figure $2 \mathrm{~A})$. In the 160 patients treated with levodopa, probability of developing any motor fluctuation five years from levodopa initiation was $30.6 \%(95 \%$ Cl 22.6-40.7\%). In 191 patients (including eight untreated patients) the probability of developing motor fluctuations five years from diagnosis was $23.4 \%$ (95\% Cl 16.6-30.4\%).The factors independently associated with the development of motor fluctuations in the main multivariable model were higher cumulative levodopa dose-over the four follow up since baseline, female sex, and younger age at diagnosis (Table 1). In the-secondary analyses the presence of tremor at diagnosis was associated with a higher rate and moderate (though not high) lifetime caffeine intake was associated with a lower rate of motor fluctuations. Wone the variables was significantly associated in multivariable analyses.

Table 1. Characteristics of patients who developed motor fluctuations 


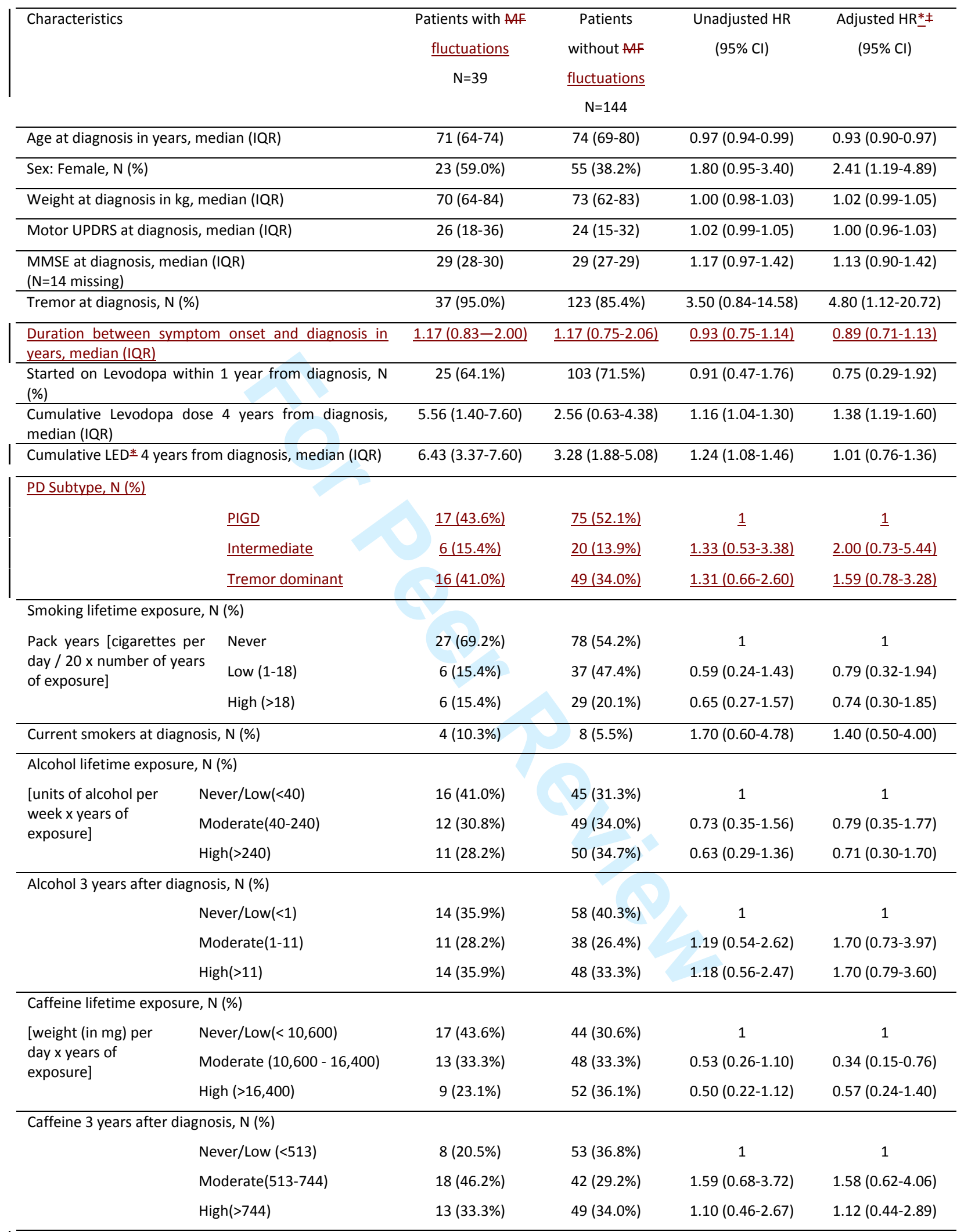

*Cumulative levodopa-equivalent dose (LED) up to 4 years from diagnosis (mg ILevodopa-equivalent dose (mg) * number of days of treatment $x 10^{-5}$; one unit is equivalentequals about to $70 \mathrm{mg}$ levodopa on average a day for 4 years). $\neq$ Variables adjusted for the variables in the final multivariable model (age at diagnosis, sex, motor UPDRS at diagnosis, and ç Eumulative ltevodopa dose 4 years from diagnosis). Abbreviations:HR=hazards ratio; IQR=interquartile range $; \mathrm{PIGD}=$ postural instability and gait disorder: $\mathrm{MF}=$ motor fluctuations. 


\section{Dyskinesias}

52 patients (28.4\%) had-developed dyskinesias by the time of data extraction-(see table 2 and figure 2B). At onset, only three patients ( $1.6 \%$ of total participants, $5.8 \%$ of those with dyskinesia) rated their dyskinesias as painful and five (2.7\% of total participants, $9.6 \%$ of those with dyskinesia) as mildly disabling; the rest were not disabling. Only 8 patients ( $4.4 \%$ of total participants, $15.4 \%$ of those with dyskinesia) developed dyskinesias which required treatment changes (such as decrease of levodopa dose or addition of amantadine). The median dyskinesia-free survival time to of dyskinesias-was 85 months. After five years on therapy the-Kaplan-Meier estimates of the probability of developing dyskinesias after five years, with different baselines for time measurement, are shown in table 3. was 37.0\% (95\% Cl 28.5-47.1\%) and of developing severe dyskinesias was $4.0 \%$ (95\% Cl 1.5-10.4\%) (Figure 2B). The median time to onset of dyskinesias was 85 months. In the 160 patients treated with levodopa, probability of developing any dyskinesia five years from levodopa initiation was $43.6 \%$ (95\% Cl 33.7-54.9). In 191 patients (including eight untreated patients) the probability of developing dyskinesias five years from diagnosis was $29.3 \%(95 \% \mathrm{Cl} 22.5-37.6 \%)$. Higher cumulative levodopa dose at 4 years from diagnosis-and ${ }_{7}$ female sex were found to be independent risk factors for the development of dyskinesias in the main multivariable-model (Table 2). In the additional models, symptom duration and the presence of tremor at diagnosis was-were associated with higher fates ofmore dyskinesias and there was a suggestion that higher MMSE score was associated with increased risk.

Table 2. Characteristics of patients who developed dyskinesias

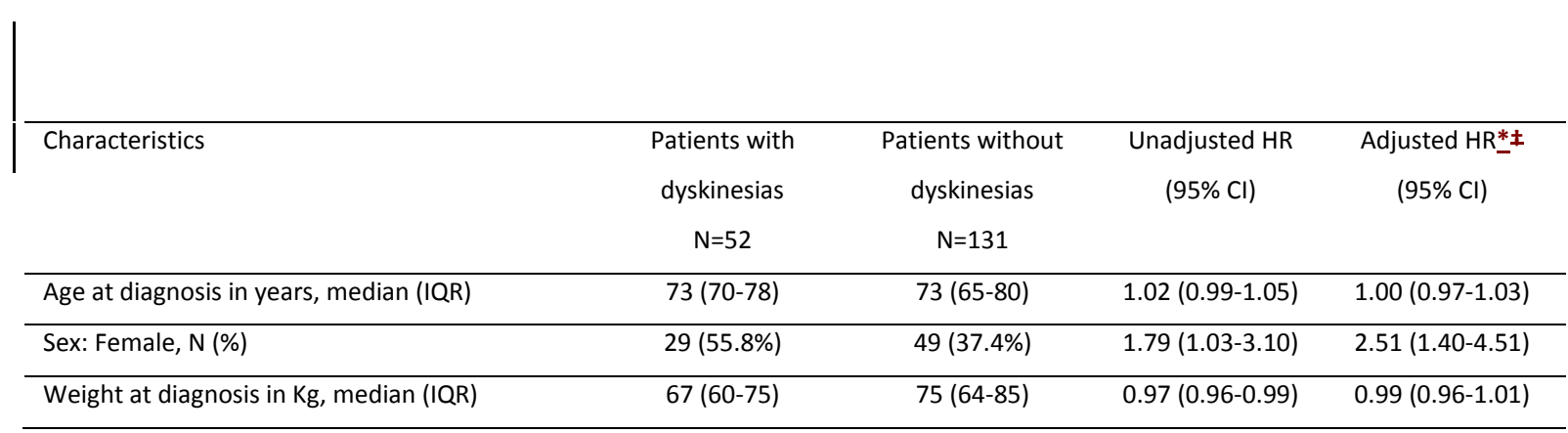




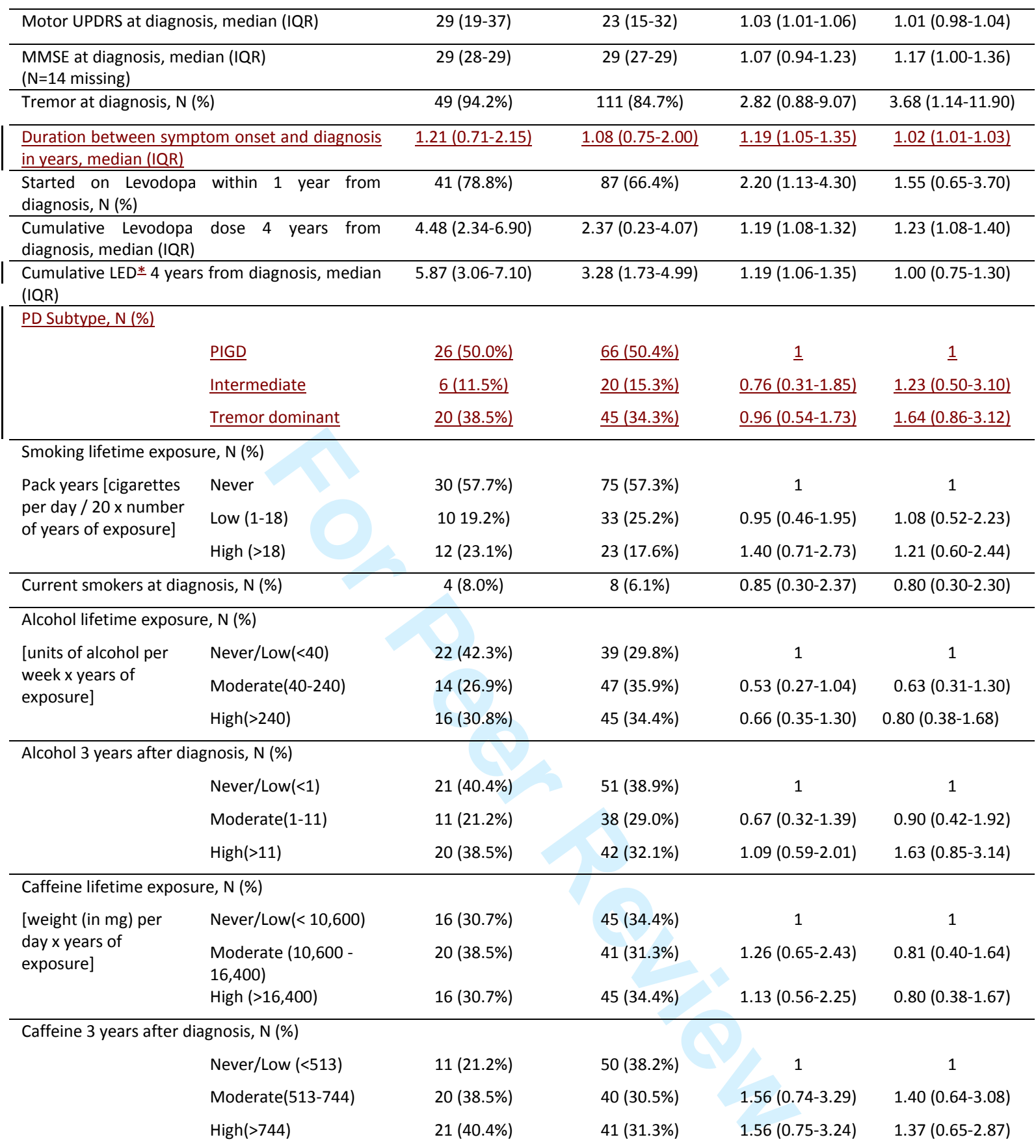

* Cumulative levodopa-equivalent dose (LED) up to 4 years from diagnosis ( $m$ I Hevodopa-equivalent dose_* number of days of treatment $x 10^{-5} ;$ one unit is equivalent to $70 \mathrm{mg}$ levodopa on average a day for 4 years). $\mp$ Variables are adjusted for the variables in the final multivariable model (age at diagnosis, sex, motor UPDRS

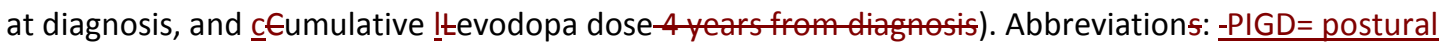
instability and gait disorder.

Table 3: Kaplan-Meier probabilities of developing motor fluctuations and dyskinesias after five years from dopaminergic treatment initiation, from levodopa initiation, and from diagnosis. 


\begin{tabular}{|lcc}
\hline \multicolumn{1}{c}{ Starting dopaminergic treatment $(\mathrm{N}=183)$} & $\underline{29.2 \%(21.5-38.8)}$ & $\underline{37.0 \%(28.5-47.1)}$ \\
\hline$\quad$ Starting levodopa treatment $(\mathrm{N}=160)$ & $\underline{30.6 \%(22.6-40.7)}$ & $\underline{43.6 \%(33.7-54.9)}$ \\
\hline$\frac{23.42 .8 \%(16.67-30.47)}{\text { Diagnosis }(\mathrm{N}=-189)}$ & $\underline{29.36 \%(22.57-37.68)}$ \\
\hline $\begin{array}{l}\text { Severe complications } \\
\frac{19.8 \%(13.4-28.8)}{4.0 \%(1.5-10.4)}\end{array}$ & & \\
\hline$\underline{\text { Starting dopaminergic treatment }(\mathrm{N}=183)}$ & $\underline{19.8 \%(13.4-28.8)}$ & $\underline{4.0 \%(1.5-10.4)}$ \\
\hline$\underline{95 \% \text { confidence intervals are in parentheses. }}$ & & \\
\hline
\end{tabular}

\section{Motor fluctuations and dyskinesias}

23 patients (12.6\%) had-developed both fluctuations and dyskinesias-by the time of data extraction, of whom 14 had developed dyskinesias first.

\section{DISCUSSION}

We have estimated that aAbout $30 \%$ of patients with PD developed motor fluctuations and about $37 \%$ developed dyskinesias within five years of starting dopaminergic treatment. These were higher risks than described in two other community-based incidence cohorts that examined the development of dyskinesias. A Mayo Clinic series from the Mayo Clinic, between 1976 and 1990 reported that $30 \%$ of patients developed dyskinesias of any severity were present in $30 \%$ of patients by five years of starting levodopa ${ }^{8}$ and in an incident cohort in Cambridge, UK, another study- fewer than $20 \%$ of patients developed dyskinesias five years from diagnosis. ${ }^{7}$ The reasons for these differences, between similar studies, are unclear. No previous community-based inception studies have reported the frequency of motor fluctuations. We found complications to be less common than several other studies, some of which were older and therefore used higher levodopa doses than are is-used in current practice. ${ }^{4,18}$ Dyskinesias were more common in our cohort than motor fluctuations, similar to some studies ${ }^{15,28}$ but not others. ${ }^{16,12,18}$ Disabling dyskinesias were rare and ${ }_{2}$ although the majority ofmost patients with motor fluctuations did have-need changes in treatment ${ }_{L}$-due to their development, only a small minorityvery few $(4 \%)$ developed dyskinesias necessitating treatment 
changes in treatment $(4 \%$ at 5 years). This is lower than one previous incident study in which reported that $17 \%$ of patients required treatment changes developed-for dyskinesias that required treatment changes by-within five years of levodopa treatment withinitiation-levodopa. ${ }^{8}$ That study used patient records between 1976 and 1990 and the difference inlower incidence of severe dyskinesias in our study, could also-be due to lower levodopa doses used in more recent prescribing practice.

Levodopa exposure has consistently been reported as the strongest risk factor for the development of motor fluctuations and dyskinesias in observational studies, ${ }^{4,8,16}$ randomised trials of levodopa versus dopamine agonists ${ }^{29,30}$ and randomised trials of different levodopa doses. ${ }^{31}$ Previous studies have investigated several measures of levodopa exposure, including the initial average daily dose; ${ }^{7}$ average daily LED; ${ }^{8}$ or levodopa dose at onset of dyskinesias (or end study end in those without dyskinesias). ${ }^{12}$ Here we have compared cumulative both levodopa only and; levodopa-equivalent doses up to four years and early (within one year of diagnosis) commencement of levodopa. Although these variables were correlated, only cumulative levodopa dose was significantly associated with motor complications when they were entered into a multivariable model together. This suggests that there is little additional effect of non-levodopa dopaminergic treatments, which is similar to findings from a recent meta-analysis. ${ }^{32}$ It also suggests there is no absolute requirement to avoid early levodopa treatment in PD but, as others have suggested, ${ }^{12}$ it is important to use the smallest dose that adequately controls the patient's symptoms throughout the course of PD. The fact that baseline motor severity (UPDRS) was not an independent risk factor for motor complications suggests that the association between cumulative levodopa dose and motor complications is not just due to worse disease severity with greater dopaminergic neuronal loss in those needing more levodopa.It can also be argued that levodopa dose is a proxy for disease severity, so that those with more aggressive disease are both more likely to get motor complications and to receive more levodopa. However, Oour data showed that baseline motor was not an 
independent risk factor, although we may have been underpowered to detect this, which suggests this finding is not confounded by disease severity. This is in By contrast to This conflicts with -one large trial which found that both baseline disease severity and levodopa doses were associated with higher frequency of motor complications. ${ }^{12}$ However, eEvidence that levodopa increases risk of dyskinesias independently of disease severity also comes from randomised clinical trials, in which disease severity is randomly allocated to each arm, and those with higher doses of levodopa ${ }^{31}$ or levodopa and entacapone ${ }^{33}$ had higher incidence of dyskinesias.

Many previous studies have shown a relationship betweenthat younger age at PD-onset and developing motor complicationsis associated with more, in particular dyskinesias, ${ }^{9,10}$ and it was the most powerful predictor in a recent large trial. ${ }^{12}$ We found that age at diagnosis is an independent risk factor for developing motor fluctuations, but notdid not predict dyskinesias. This latter, similar to finding supports results from-another community--based incidence study that showed that age at baseline was not a risk factor for dyskinesias. ${ }^{7}$ It may be that, as a community-based incidence study, with proportionally few young-onset patients (4.4\% under 50$)$, there was insufficient power to detect an effect of age on dyskinesias.

Duration between symptom onset and diagnosis was found to be an independent risk factor for developing dyskinesias but not motor fluctuations. This result was independent of baseline disease severity and levodopa dose so does not appear to be an effect of those presenting later being treated with higher doses of levodopa. This finding must be interpreted cautiously because it was a secondary analysis and the variable is subject to recall bias but it may suggests that patients that with more indolent onset of their PD symptoms may be at a higher risk of developing dyskinesias.

The presence of tremor at diagnosis was associated with higher risk of both motor fluctuations and dyskinesias in the secondary analyses, which contrasts with a previous smaller study. ${ }^{34}$ Although this 
Female gender was an independent risk factor for both motor fluctuations and dyskinesias, as previous studies have also shown. ${ }^{12}$ - The reasons for these-gender differences in the development of motor complications are unclear. A possible explanation is that -lower average weight in females results in higher levodopa doses per body weight, and some previous studies found lower weight was a risk factor for dyskinesias. ${ }^{357,368}$ We did not, however, demonstrate an association with baseline weight but changes in weight after diagnosis may be more important in the development of complications. It has also been suggested that females have a reduced genetic protection from a dopamine receptor polymorphism, ${ }^{11}$ and hormonal differences may be important, with evidence from animal models of effects of oestrogen on the basal ganglia. ${ }^{3790}$

Moderate lifetime caffeine exposure was associated with a reduced risk of developing motor fluctuations in the secondary multivariable analyses but there was no dose-response gradient and, given the large number of associations tested in the secondary analyses, it may well be a false positive. Nevertheless, Observational data from a clinical trial showed caffeine was associated with a lowered risk of dyskinesias ${ }^{20}$ and a elinical-trial of another adenosine A2A antagonist, iłstradefylline reduced daily OFF time. ${ }^{21}$ Moderate lifetime caffeine exposure was associated with a reduced risk of developing motor fluctuations in the secondary multivariable analyses but there was no doseresponse gradient and, given the large number of associations tested in the secondary analyses, it may well be a false positive. Similarly, we did not find-caffeine, smoking or alcohol exposure, either before or after diagnosis to be associated with lower risk of dyskinesias. This is in lineconsistent with a previous study which showed smoking was not associated with motor complications. ${ }^{14}$ However, we did not havelacked power to identify a-small effects of these 
exposures on motor complications; data was collected retrospectively for pre-diagnostic exposure; and there were very few current smokers in the cohort.

The principal strengths of this study are its prospective design; the representative sample (attempts were made ito identify all patients in the community with a new diagnosis of PDparkinsonian syndrome); that diagnoses were-regularly reviewed of diagnoses to achieve high-improve diagnostic accuracy; that patients clinicallyfrequent (at least yearly) clinical review to obtain data on motor complications; very high study retention; and careful statistical analyses. Additionally, cumulative levodopa doses were calculated up to 4 years rather than at complication onset. This is clearly better than comparing levodopa dose at onset of motor complications with levodopa dose at end of study in those without complications as this is confounded by difference in time.

The study has several limitations. Firstly, study size, while not small in terms of previous studies of the development of -motor complications, is not large enoughinsufficient to identify weak associations or investigate interactions. Secondly, average follow-up duration was only about five years, so better data may be obtained with longer follow-up. Thirdly, eumulative levodopa-doses were calculated up to 4 years and not at complication onset. However, identifying a time-point for control comparisons would be difficult if this was done and thThis is clearly better than comparing levodopa dose at onset of motor complications with levodopa dose at end of study in those without complications as this is clearly confounded by difference in time. Fourthly, some inaccuracy in defining onset of complications is inevitable. Exact timing of onset of both dyskinesias and motor fluctuations was mostly subject to patient recall, although some patients' dyskinesias were observed at clinic visits before they were noticed by the patients themselves, and were recorded as starting when seen. Thus the time to onset of dyskinesias may be overestimated. Assessment of severity was based on data about changes in therapy, which was derived from comprehensive clinical letter that invariably included reasons for treatment changes so we believe this was a reliable assessment. 


\section{1}

Fourthly, the secondary analyses must be considered as exploratory as many variables were inded examined and the associations identified may therefore betype I errors are possible-false positives. Fifthly, data on caffeine, smoking and alcohol were partly retrospective, only average exposures were used, and we did not gather data on sources of caffeine other than tea and coffee.

In conclusion, we are the first to describe the development of both motor fluctuations and dyskinesias in a representative, community-based, incident cohort of PD. We estimate that $29 \%$ and $37 \%$ develop motor fluctuations and dyskinesias respectively after 5 years of dopaminergic treatment. Dyskinesias requiring treatment changes were rare ( $4 \%$ at 5 years), which is lower than

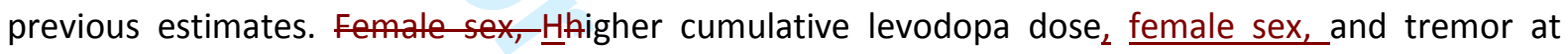
diagnosis were independent risk factors for both motor complications; and-moderate lifetime caffeine exposure and younger age fithfor fewer motor fluctuations; and longer pre-diagnosis symptom duration for more dyskinesias. Further work with more patients with longer follow-up would be useful to obtainfor more detailed analysis of the-risk factors-associated with development of motor complications. Individual-patient-data meta-analysis of existing representative studies would be an efficient way to do this.

\section{ACKNOWLEDGEMENTS}

We acknowledge funding for the PINE study from Parkinson's UK, the Scottish Chief Scientist Office, the BMA Doris Hillier award, RS Macdonald Trust, the BUPA Foundation, NHS Grampian endowments and SPRING. We thank the patients for their participation and the research staff who collected data and supported the study database.

\section{AUTHOR'S ROLES}


The authors contributed to the following specific roles in the project and manuscript preparation as indicated:

1. Research project: A. Conception, B. Organization, C. Execution;

2. Statistical Analysis: A. Design, B. Execution, C. Review and Critique;

3. Manuscript Preparation: A. Writing of the first draft, B. Review and Critique

Nicholas W Scott (1B, 1C, 2B, 2C, 3A)

Angus D Macleod (1B, 2A, 2B, 2C, 3B)

Carl E Counsell (1A, 1B, 2C, 3B)

\section{FINANCIAL DISCLOSURE}

Nicholas W Scott: none

Angus D Macleod: Funded by a Clinical Academic Fellowship from the Scottish Chief Scientist Office. Also received research funding from Parkinson's UK.

Carl E Counsell: Research funding from Parkinson's UK, Scottish Chief Scientist Office, National Institute of Health Research, and Engineering and Physical Sciences Research Council

\section{REFRENCES}

1. Samii A, Nutt JG, Ransom BR. Parkinson's disease. Lancet 2004; 363 (9423):1783-1794.

2. Chapuis S, Ouchchane L, Metz O, Gerbaud L, Durif F. Impact of the motor complications of Parkinson's disease on the quality of life. Movement Disorders 2005; 20 (2): 224-230. 
3. Marras C, Lang A, Krahn M, Tomlinson G, Naglie G. Parkinson Study Group, Quality of life in early Parkinson's disease. Movement Disorders 2004; 19 (1): 22-28.

4. Ahlskog JE, Muenter MD. Frequency of levodopa-related dyskinesias and motor fluctuations as estimated from the cumulative literature. Movement Disorders 2001; 16 (3): 448-458.

5. Rybicki BA, Johnson CC, Gorell JM. Demographic differences in referral rates to neurologists of patients with suspected Parkinson's disease: implications for case-control study design. Neuroepidemiology 1995; 14 (2): 72-81.

6. Fitzsimmons PR, Blayney S, Mina-Corkill S, Scott GO. Older participants are frequently excluded from Parkinson's disease research. Parkinsonism Related Disorders 2012; 18 (5): 585-589.

7. Evans JR, Mason SL, Williams-Gray CH, et al. The natural history of treated Parkinson's disease in an incident, community based cohort. J Neurology Neurosurgery Psychiatry 2011; 82 (10): 11121118.

8. Van Gerpen JA, Kumar N, Bower JH, Weigand S, Ahlskog JE. Levodopa-associated dyskinesia risk among Parkinson disease patients in Olmsted County, Minnesota, 1976-1990. Archives Neurology 2006; 63 (2): 205-209.

9. Kumar N, Van Gerpen JA, Bower JH, Ahlskog JE. Levodopa-dyskinesia incidence by age of Parkinson's disease onset. Movement Disorders 2005; 20 (3): 342-434.

10. Ku S, Glass GA. Age of Parkinson's disease onset as a predictor for the development of dyskinesia. Movement Disorders 2010; 25 (9): 1177-1182.

11. Zappia M, Annesi G, Nicoletti G, et al. Sex Differences in clinical and genetic determinants of levodopa peak-dose dyskinesias in Parkinson disease: an exploratory study. Archives Neurology 2005; 62 (4): 601-605.

12. Olanow CW, Kieburtz K, Rascol O, et al. Factors Predictive of the Development of LevodopaInduced Dyskinesia and Wearing-Off in Parkinson's Disease. Movement Disorders 2013; 28 (8): 1064-1071. 
13. Rascol O, Brooks DJ, Korczyn AD, et al. Development of dyskinesias in a 5-year trial and ropinirole and L-dopa. Movement Disorders 2006; 21 (11): 1844-1850.

14. Hassin-Baer S, Molchadski I, Cohen OS, et al. Gender effect on time to levodopa-induced dyskinesias. Journal of Neurology 2011; 258 (11): 2048-2053.

15. Garcia-Ruiz PJ, del Val J, Fernandez IM, Herranz A. What factors influence motor complications in Parkinson disease?: A 10-year prospective study. Clinical Neuropharmacology 2012; 35 (1):1-5.

16. Schrag A, Quinn N. Dyskinesias and motor fluctuations in Parkinson's disease: a communitybased study. Brain 2000; 123 (11): 2297-2305.

17. Jankovic J. Motor fluctuations and dyskinesias in Parkinson's disease: clinical manifestations. Movement Disorders 2005; 20 (Suppl. 11): S11-16.

18. Hauser RA, McDermott MP, Messing S. Factors associated with the development of motor fluctuations and dyskinesias in Parkinson disease. Archives Neurology 2006; 63 (12): 1756-1760.

19. Noyce AJ, Bestwick JP, Silveira-Moriyama, et al. Meta-analysis of early nonmotor features and risk factors for Parkinson disease. Annals Neurology 2012; 72 (6): 893-901.

20. Wills AM, Eberly S, Tennis M, et al. Caffeine consumption and risk of dyskinesia in CALM-PD. Movement Disorders 2013; 28 (3): 380-383.

21. Mizuno $Y$, Kondo $T$, Japanese Istradefylline study group. Adenosine A2a receptor antagonist Istradefylline reduces daily OFF time in Parkinson's disease. Movement Disorders 2013; 28 (8): $1138-1141$.

22. Taylor KS, Counsell CE, Harris CE, Gordon JC, Smith WC. Pilot study of the incidence and prognosis of degenerative Parkinsonian disorders in Aberdeen, United Kingdom: methods and preliminary results. Movement Disorders 2006; 21 (7): 976-982.

23. Caslake R, Scott N, Gordon J, et al. Age-, gender-, and socioeconomic status-specific incidence of Parkinson's disease and parkinsonism in North East Scotland: The PINE study. Parkinsonism Related Disorders 2013; 19 (5): 515-521. 
24. Hughes AJ, Daniel SE, Kilford L, Lees AJ. Accuracy of clinical diagnosis of idiopathic Parkinson's disease: a clinico-pathological study of 100 cases. J Neurology Neurosurgery Psychiatry 1992; 55

(3): 181-184.

24.25. Tomlinson CL, Stowe R, Patel S, Rick C, Gray R, Clarke CE. Systematic review of levodopa dose equivalency reporting in Parkinson's disease. Movement Disorders 2010: 25 (15): 26492653.

25.26. U.S. Department of Agriculture, Agriculture Research Service. USDA National Nutrient Database for Standard Reference, Release 24. 2011.

26.27. Peduzzi P, Concato J, Feinstein AR, Holford TR. Importance of events per independent variable in proportional hazards regression analysis, II: accuracy and precision of regression estimates. Journal of Clinical Epidemiology 1995; 48 (12): 1503-1510.

27.28. Mazzella L, Yahr MD, Marinelli L, Huang N, Moshier E, Di Rocco A. Dyskinesias predict the onset of motor response fluctuations in patients with Parkinson's disease on I-dopa monotherapy, Parkinsonism Related Disorders 2005; 11 (3): 151-155.

28.29. Parkinson study Group. Pramipexole vs levodopa as initial treatment for Parkinson's disease. A randomised controlled trial. JAMA 2000; 284 (15): 1931-1938.

29-30. Parkinson Study Group CALM Cohort Investigators. Long-term effect of initiating pramipexole vs levodopa in early Parkinson disease. Archives Neurology 2009; 66 (5): 563-570.

30.31. Fahn S, Oakes D, Shoulson I, et al. Levodopa and the progression of Parkinson's disease. New England Journal of Medicine 2004; 351 (24): 2498-2508.

31.32. Chondrogiorgi M, Tatsioni A, Reichmann $H$ and Konitsiotis S. Dopamine agonist monotherapy in Parkinson's disease and potential risk factors for dyskinesia: a meta-analysis of levodopa controlled trials. European Journal of Neurology 2014; 21 (3): 433-440.

32.33. Stocchi F, Rascol O, Kieburtz K, et al. Initiating levodopa/carbidopa therapy with and without entacapone in early Parkinson disease. The STRIDE-PD study. Annals of Neurology 2010; 68 (1): $18-27$. 
33.34. Kipfer S, Stephan MA, Schüpbach WM, Ballinari P, Kaelin-Lang A. Resting tremor in Parkinson disease: a negative predictor of levodopa-induced dyskinesia. Archives Neurology 2011; 68 (8): 1037-1039.

34.35. Sharma JC, Macnamara L, Hasoon M, Vassallo M, Ross IN. Cascade of levodopa dose and weight related dyskinesia in Parkinson's disease (LD-WD-PD cascade). Parkinsonism Related Disorders 2006; 12 (8): 499-505.

36. Sharma JC, Ross IN, Rascol O, Brooks D. Relationship between weight, levodopa and dyskinesia: the significance of levodopa dose per kilogram body weight. European Journal of Neurology 2008; 15 (5): 493-496.

35-37. Smith KM, Dahodwala, N. Sex differences in Parkinson's disease and other movement disorders. Experimental Neurology 2014; 259: 44-56.

\section{FIGURE LEGENDS}

Figure 1. Flow chart of patients included in the analysis and initiation of levodopa treatment. FU: Follow-Up.

*Patients received only dopamine agonist or MAO-B inhibitors within 4 years of follow-up, except for 5 patients who received treatment after 4 years of follow-up.

Figure 2. Kaplan-Meier estimates of the probability that PD patients on a dopaminergic therapy will be free from (A) motor fluctuations and (B) dyskinesias. -In each graph, the blue line represents the development of any motor complication and the red represents the development of severe complications, i.e., those necessitating needing changes to treatment. 
Recruited parkinsonian patients

Other parkinsonism

Declined FU

10

Died before first FU

6

Not on dopaminergic therapy

183

Idiopathic PD patients analysed

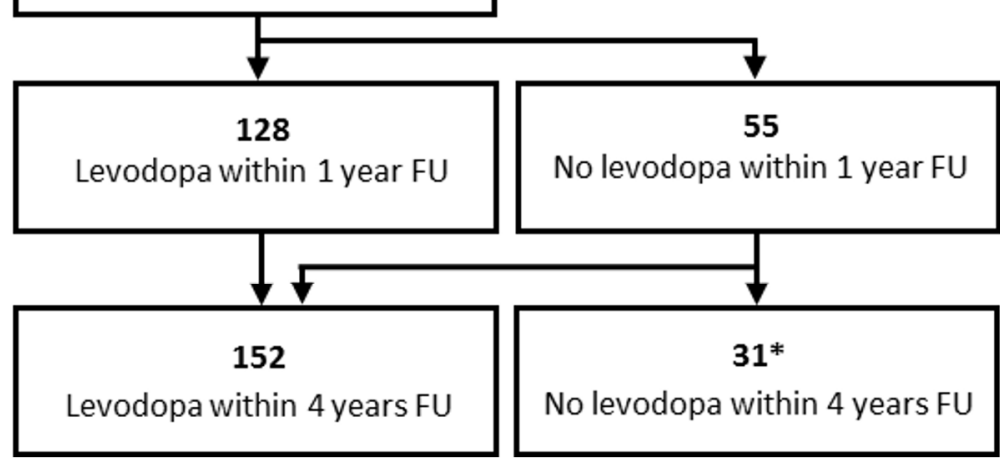

$201 \times 316 \mathrm{~mm}(300 \times 300$ DPI) 
Title: Motor complications in an incident Parkinson's disease cohort

Manuscript ID: EJoN-15-0093 Author(s): Scott, Nicholas; Macleod, Angus; Counsell, Carl

We would like to thank the reviewers for their helpful comments and include a detailed reply to individual comments, in red, below.

Reviewer: 1

COMMENTS TO AUTHOR(S)

The present investigation drwas its major strength from its cohort based, incident-case methods. The theme is suitable for EJN and of clinical relevance, as it adresses one of the major concerns when manging tretment options for Parkinsons Disease Patients. There are, however, one point that deserve better explanation. The authors should be more precise regarding the type of dopaminergic tretament other than levo-dopa. How many patientes were on ropinierol, comt inhibitors, parmaipexeole and son on.

Response to Reviewer: 1

We have inserted the following explanation into the text (page 7, lines 4-7):

"The patients who received dopaminergic therapy in the first four years, but not levodopa, were mostly treated with ropinirole (33 patients), pramipexole (17 patients), selegiline (13 patients), COMT inhibitors (2 patients), rasagiline (1 patient), or ergot-derived dopamine agonists ( 2 patients)."

Reviewer: 2

COMMENTS TO AUTHOR(S)

1. Did the authors collect information on the time between clinical onset of disease and diagnosis?

This information was collected but not analysed. In response to the reviewer's comment we have performed unadjusted and adjusted analysis of time between clinical onset and diagnosis. Longer duration between symptom onset and diagnosis was independently associated with risk of dyskinesias. The following changes have therefore been made:

Inserted into the Abstract (p. 2, lines 22-23): "longer symptom duration"

Inserted into the Methods (p.6, lines 9-10): "symptom duration prior to diagnosis,"

Inserted into Results Tables 1 (p.8) and 2 (p.9).

Inserted into Discussion (p.12, lines 15-20): "Duration between symptom onset and diagnosis was found to be an independent risk factor for developing dyskinesias but not motor fluctuations. This result was independent of baseline disease severity and levodopa dose so does not appear to be an effect of those presenting later being treated with higher doses of levodopa. This finding must be interpreted cautiously because it was a secondary analysis and the variable is subject to recall bias 
but it may suggest that patients with more indolent onset of their PD symptoms may be at a higher risk of developing dyskinesias."

Inserted into Discussion (p.17, line 14): "and longer pre-diagnosis symptom duration for more dyskinesias"

2. What was the percentage of change of diagnosis from baseline over follow up in this cohort?

We have inserted the following comment into text to add this information Methods (p.4 line 13-14):

"15\% of patients with a latest diagnosis of PD had initially had an alternative diagnosis."

3. How reliable was the assessment of severe motor complication; onset of dyskinesias and motor fluctuations?

We have added the following sentences into the Discussion (p.14 lines 7-13) to discuss the potential limitation of inaccuracies, particularly in identifying the timing of onset of these complications.

"Thirdly, some inaccuracy in defining onset of complications is inevitable. Exact timing of onset of both dyskinesias and motor fluctuations was mostly subject to patient recall, although some patients' dyskinesias were observed at clinic visits before they were noticed by the patients themselves, and were recorded as starting when seen. Thus the time to onset of dyskinesias may be overestimated. Assessment of severity was based on data about changes in therapy, which was derived from comprehensive clinical letter that invariably included reasons for treatment changes so we believe this was a reliable assessment."

4. It would be interesting to know why eight patients did not start levo-dopa or any dopaminergic agent. Were they different from the others?

We have checked the case record for these patients and added the relevant data to the text. While doing this, we have discovered an error in the flow chart relating only to patients not included in the main analysis. We have updated figure 1 with the correct data. Those who were not treated were probably more likely to be tremor dominant in their presentation, but we have not made a formal comparison due to low numbers in this group. Their ages were similar. We have added the following reasons for patients not starting treatment into the Results section (p.6, lines 22-25):

"These patients (mean age 70.8) were not treated because they had mild disease with tremor dominance $(N=3)$, had early dementia while motor symptoms were still mild $(N=1)$, refused treatment $(\mathrm{N}=1)$, or did not tolerate levodopa $(\mathrm{N}=1) . "$

\section{What was the median duration of folow-up?}


The median Follow-Up 60 months, IQR (48 months - 72 months). As this was very similar to the mean duration of follow-up (59 months, SD 22 months), which is stated in the text (page 7) we have not added the median follow-up to the manuscript.

6. Did the authors distinguish in the analyses tremor dominant from akinetic phenotype with gait disturbances? This is of course different from presence of tremor at onset.

We have added this data into the Results Tables 1 (p.8) and 2 (p.9). There was no significant effect of PD subtype on the risk of developing either motor fluctuations or dyskinesias.

\section{The authors should develop more the discussion on sex differences.}

We have expanded this section of the discussion, so that it now reads ( $p .13$, lines 1-9):

"Female gender was an independent risk factor for both motor fluctuations and dyskinesias, as previous studies have also shown. ${ }^{12}$ The reasons for gender differences in the development of motor complications are unclear. A possible explanation is that lower average weight in females results in higher levodopa doses per body weight, and some previous studies found lower weight was a risk factor for dyskinesias. ${ }^{35,36}$ We did not, however, demonstrate an association with baseline weight but changes in weight after diagnosis may be more important in the development of complications. It has also been suggested that females have a reduced genetic protection from a dopamine receptor polymorphism, ${ }^{11}$ and hormonal differences may be important, with evidence from animal models of effects of oestrogen on the basal ganglia. ${ }^{37 "}$ 
Table 1. Characteristics of patients who developed motor fluctuations

\begin{tabular}{|c|c|c|c|}
\hline Patients with & Patients & Unadjusted HR & Adjusted HR* \\
\hline fluctuations & without & $(95 \% \mathrm{Cl})$ & $(95 \% \mathrm{Cl})$ \\
\hline \multirow[t]{2}{*}{$N=39$} & fluctuations & & \\
\hline & $\mathrm{N}=144$ & & \\
\hline
\end{tabular}

\begin{tabular}{|c|c|c|c|c|c|}
\hline \multicolumn{2}{|c|}{ Age at diagnosis in years, median (IQR) } & $71(64-74)$ & $74(69-80)$ & $0.97(0.94-0.99)$ & $0.93(0.90-0.97)$ \\
\hline \multicolumn{2}{|l|}{ Sex: Female, N (\%) } & $23(59.0 \%)$ & $55(38.2 \%)$ & $1.80(0.95-3.40)$ & $2.41(1.19-4.89)$ \\
\hline \multicolumn{2}{|c|}{ Weight at diagnosis in kg, median (IQR) } & $70(64-84)$ & $73(62-83)$ & $1.00(0.98-1.03)$ & $1.02(0.99-1.05)$ \\
\hline \multicolumn{2}{|c|}{ Motor UPDRS at diagnosis, median (IQR) } & $26(18-36)$ & $24(15-32)$ & $1.02(0.99-1.05)$ & $1.00(0.96-1.03)$ \\
\hline \multicolumn{2}{|c|}{$\begin{array}{l}\text { MMSE at diagnosis, median (IQR) } \\
\text { ( } N=14 \text { missing) }\end{array}$} & $29(28-30)$ & $29(27-29)$ & $1.17(0.97-1.42)$ & $1.13(0.90-1.42)$ \\
\hline \multicolumn{2}{|c|}{ Tremor at diagnosis, $\mathrm{N}(\%)$} & $37(95.0 \%)$ & $123(85.4 \%)$ & $3.50(0.84-14.58)$ & $4.80(1.12-20.72)$ \\
\hline \multicolumn{2}{|c|}{$\begin{array}{l}\text { Duration between symptom onset and diagnosis in } \\
\text { years, median (IQR) }\end{array}$} & $1.17(0.83-2.00)$ & $1.17(0.75-2.06)$ & $0.93(0.75-1.14)$ & $0.89(0.71-1.13)$ \\
\hline \multicolumn{2}{|c|}{$\begin{array}{l}\text { Started on Levodopa within } 1 \text { year from diagnosis, N } \\
(\%)\end{array}$} & $25(64.1 \%)$ & $103(71.5 \%)$ & $0.91(0.47-1.76)$ & $0.75(0.29-1.92)$ \\
\hline \multicolumn{2}{|c|}{$\begin{array}{l}\text { Cumulative Levodopa dose } 4 \text { years from diagnosis, } \\
\text { median (IQR) }\end{array}$} & $5.56(1.40-7.60)$ & $2.56(0.63-4.38)$ & $1.16(1.04-1.30)$ & $1.38(1.19-1.60)$ \\
\hline \multicolumn{2}{|c|}{ Cumulative LED 4 years from diagnosis, median (IQR) } & $6.43(3.37-7.60)$ & $3.28(1.88-5.08)$ & $1.24(1.08-1.46)$ & $1.01(0.76-1.36)$ \\
\hline \multicolumn{6}{|l|}{ PD Subtype, N (\%) } \\
\hline \multirow{3}{*}{\multicolumn{2}{|c|}{$\begin{array}{l}\text { PIGD } \\
\text { Intermediate } \\
\text { Tremor dominant }\end{array}$}} & $17(43.6 \%)$ & $75(52.1 \%)$ & 1 & 1 \\
\hline & & $6(15.4 \%)$ & $20(13.9 \%)$ & $1.33(0.53-3.38)$ & $2.00(0.73-5.44)$ \\
\hline & & $16(41.0 \%)$ & 49 (34.0\%) & $1.31(0.66-2.60)$ & $1.59(0.78-3.28)$ \\
\hline \multicolumn{6}{|c|}{ Smoking lifetime exposure, N (\%) } \\
\hline \multirow{3}{*}{$\begin{array}{l}\text { Pack years [cigarettes per } \\
\text { day / } 20 \times \text { number of years } \\
\text { of exposure] }\end{array}$} & Never & $27(69.2 \%)$ & $78(54.2 \%)$ & 1 & 1 \\
\hline & Low (1-18) & $6(15.4 \%)$ & $37(47.4 \%)$ & $0.59(0.24-1.43)$ & $0.79(0.32-1.94)$ \\
\hline & High $(>18)$ & $6(15.4 \%)$ & $29(20.1 \%)$ & $0.65(0.27-1.57)$ & $0.74(0.30-1.85)$ \\
\hline \multicolumn{2}{|c|}{ Current smokers at diagnosis, N (\%) } & $4(10.3 \%)$ & $8(5.5 \%)$ & $1.70(0.60-4.78)$ & $1.40(0.50-4.00)$ \\
\hline \multicolumn{6}{|c|}{ Alcohol lifetime exposure, $\mathrm{N}(\%)$} \\
\hline \multirow{3}{*}{$\begin{array}{l}\text { [units of alcohol per } \\
\text { week } x \text { years of } \\
\text { exposure] }\end{array}$} & Vever/Low $(<40)$ & $16(41.0 \%)$ & $45(31.3 \%)$ & 1 & 1 \\
\hline & Moderate(40-240) & $12(30.8 \%)$ & 49 (34.0\%) & $0.73(0.35-1.56)$ & $0.79(0.35-1.77)$ \\
\hline & $\operatorname{High}(>240)$ & $11(28.2 \%)$ & $50(34.7 \%)$ & $0.63(0.29-1.36)$ & $0.71(0.30-1.70)$ \\
\hline \multicolumn{6}{|c|}{ Alcohol 3 years after diagnosis, N (\%) } \\
\hline \multicolumn{2}{|c|}{ Never/Low $(<1)$} & $14(35.9 \%)$ & $58(40.3 \%)$ & 1 & 1 \\
\hline \multicolumn{2}{|r|}{ Moderate(1-11) } & $11(28.2 \%)$ & $38(26.4 \%)$ & $1.19(0.54-2.62)$ & $1.70(0.73-3.97)$ \\
\hline \multicolumn{2}{|c|}{$\operatorname{High}(>11)$} & $14(35.9 \%)$ & $48(33.3 \%)$ & $1.18(0.56-2.47)$ & $1.70(0.79-3.60)$ \\
\hline \multicolumn{6}{|c|}{ Caffeine lifetime exposure, N (\%) } \\
\hline \multirow{3}{*}{$\begin{array}{l}\text { [weight (in } \mathrm{mg} \text { ) per } \\
\text { day } x \text { years of } \\
\text { exposure] }\end{array}$} & Vever $/$ Low $(<10,600)$ & $17(43.6 \%)$ & $44(30.6 \%)$ & 1 & 1 \\
\hline & Moderate $(10,600-16,400)$ & $13(33.3 \%)$ & $48(33.3 \%)$ & $0.53(0.26-1.10)$ & $0.34(0.15-0.76)$ \\
\hline & High $(>16,400)$ & $9(23.1 \%)$ & $52(36.1 \%)$ & $0.50(0.22-1.12)$ & $0.57(0.24-1.40)$ \\
\hline \multicolumn{6}{|c|}{ Caffeine 3 years after diagnosis, N (\%) } \\
\hline & Vever/Low $(<513)$ & $8(20.5 \%)$ & $53(36.8 \%)$ & 1 & 1 \\
\hline & Moderate(513-744) & $18(46.2 \%)$ & $42(29.2 \%)$ & $1.59(0.68-3.72)$ & $1.58(0.62-4.06)$ \\
\hline & $\operatorname{High}(>744)$ & $13(33.3 \%)$ & 49 (34.0\%) & $1.10(0.46-2.67)$ & $1.12(0.44-2.89)$ \\
\hline
\end{tabular}

*Variables adjusted for the variables in the final multivariable model (age at diagnosis, sex, motor UPDRS at diagnosis, and cumulative levodopa dose 4). Abbreviation: PIGD= postural instability and gait disorder. 
Table 2. Characteristics of patients who developed dyskinesias

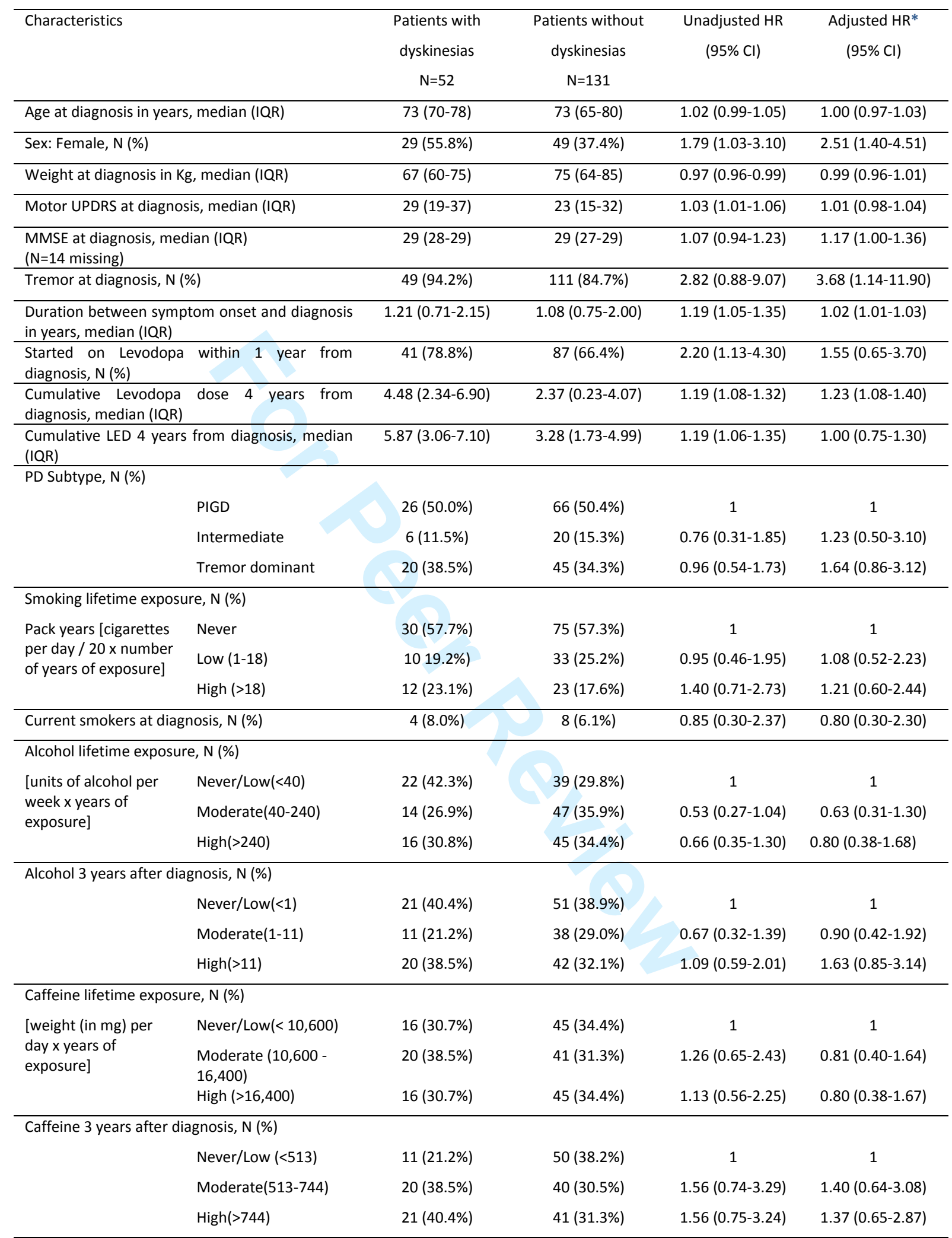

*Variables are adjusted for the variables in the final multivariable model (age at diagnosis, sex, motor UPDRS at diagnosis, and cumulative levodopa dose). Abbreviation: PIGD= postural instability and gait disorder. 
Table 3: Kaplan-Meier probabilities of developing motor fluctuations and dyskinesias after five years from dopaminergic treatment initiation, from levodopa initiation, and from diagnosis.

\begin{tabular}{lll}
\hline Baseline for time measurement & Motor fluctuations & Dyskinesias \\
\hline Any complication & & \\
\hline Starting dopaminergic treatment $(\mathrm{N}=183)$ & $29.2 \%(21.5-38.8)$ & $37.0 \%(28.5-47.1)$ \\
Starting levodopa treatment $(\mathrm{N}=160)$ & $30.6 \%(22.6-40.7)$ & $43.6 \%(33.7-54.9)$ \\
Diagnosis ( $\mathrm{N}=189)$ & $22.8 \%(16.7-30.7)$ & $29.6 \%(22.7-37.8)$ \\
\hline Severe complications & & $4.0 \%(1.5-10.4)$ \\
\hline Starting dopaminergic treatment $(\mathrm{N}=183)$ & $19.8 \%(13.4-28.8)$ & \\
\hline 95\% confidence intervals are in parentheses. &
\end{tabular}



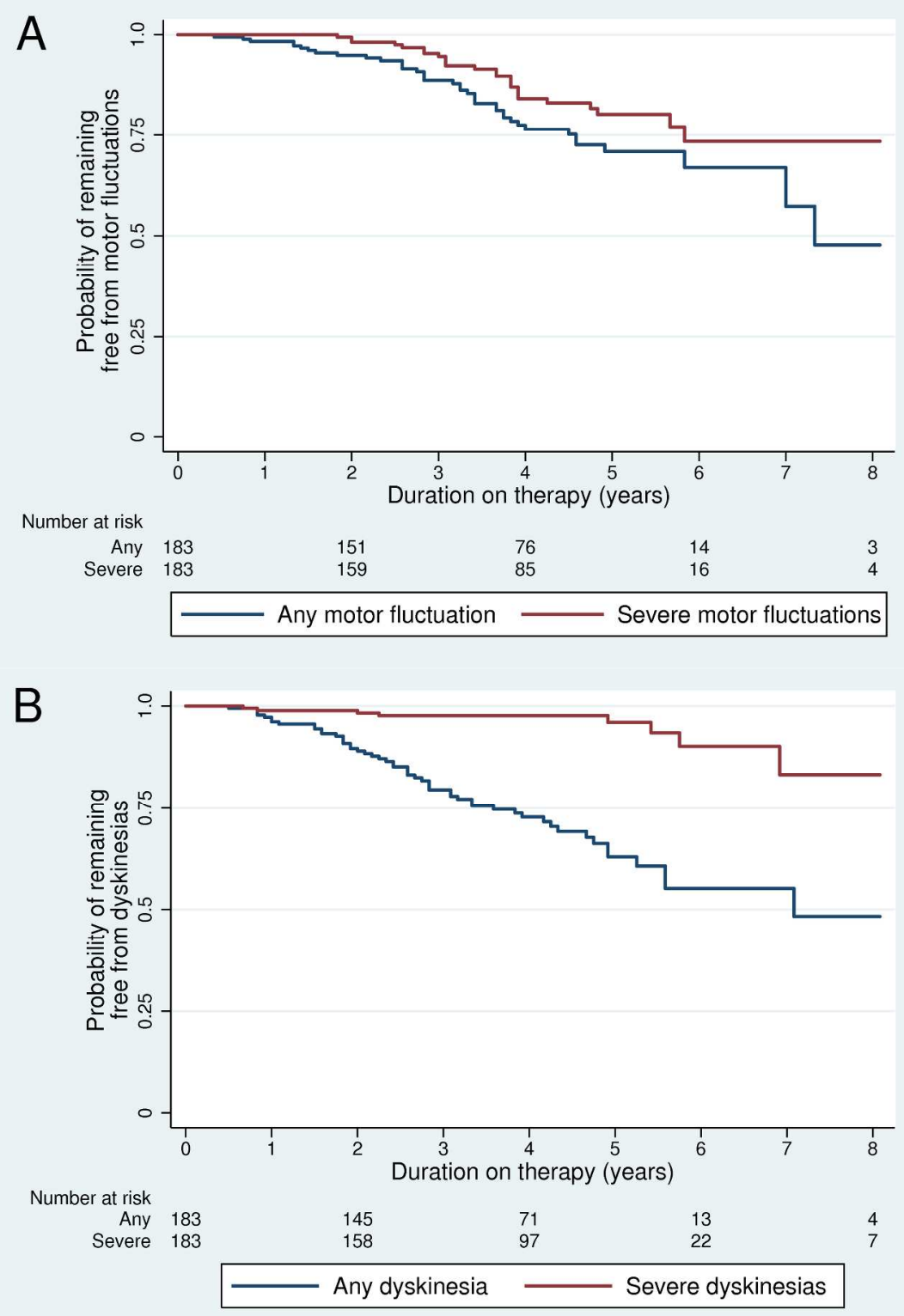

Figure 2

$203 \times 295 \mathrm{~mm}(300 \times 300 \mathrm{DPI})$ 


\section{Author Guarantee Statement}

Kindly confirm that your manuscript meets the following criteria by marking an ' $x$ ' in each appropriate box on the left. Alternatively, print the form, fill it in and scan it for submission with your manuscript files.

\begin{tabular}{|c|c|}
\hline & $\begin{array}{l}\text { The manuscript is not, either in part or whole, under active consideration by any other journal, will } \\
\text { not be submitted for review to another journal until European Journal of Neurology makes an } \\
\text { editorial decision on it, and has not been published in any other journal in either hard-copy or } \\
\text { electronic format. } \\
\text { Note: Abstracts and press reports published in connection with scientific meetings are not } \\
\text { considered as publications. }\end{array}$ \\
\hline & $\begin{array}{l}\text { All co-authors have made a substantial contribution to the design, data collection and analysis of } \\
\text { the research and the drafting of the manuscript and have reviewed and accepted the contents of } \\
\text { the manuscript prior to its submission. }\end{array}$ \\
\hline & $\begin{array}{l}\text { There has been no ghost writing by anyone not named as a co-author. The manuscript is free from } \\
\text { falsification, fabrication and plagiarism. }\end{array}$ \\
\hline & $\begin{array}{l}\text { If the study involved people, human tissues, medical records or death certificates even } \\
\text { retrospectively, the Methods/Patients section includes the full name of the institutional review } \\
\text { board or ethics committee that approved the research protocol. } \\
\text { Note: If no such approval was needed according to the local regulations, that is explained in the } \\
\text { Methods section. }\end{array}$ \\
\hline & $\begin{array}{l}\text { If the study involved a specific intervention purely for research - even a questionnaire - the } \\
\text { Methods/Patients section indicates that the study subjects or their next-of-kin gave informed } \\
\text { consent for their participation. }\end{array}$ \\
\hline & $\begin{array}{l}\text { Completed ICMJE disclosure forms for all authors are submitted along with the manuscript files. } \\
\text { The manuscript includes a section titled 'Disclosure of conflict of interest'. This section details any } \\
\text { financial relationships that the authors may have with the company whose drug or other product } \\
\text { is described in the manuscript. Where no such relationship exists, the section indicates 'None'. }\end{array}$ \\
\hline & $\begin{array}{l}\text { If the research being reported has received funding or other outside support, the manuscript } \\
\text { includes a section titled 'Acknowledgements'. This section details the sources of funding and } \\
\text { acknowledges the outside support. }\end{array}$ \\
\hline & $\begin{array}{l}\text { If the manuscript reports the results of a randomized clinical trial, the details of trial registration } \\
\text { are given in a section titled 'Trial registration'. }\end{array}$ \\
\hline & Optional comments: \\
\hline & $\begin{array}{l}\text { Name/signature of the guarantor: } \\
\qquad \text { NIKHOLAS Scott }\end{array}$ \\
\hline
\end{tabular}

